\title{
Economic analyses of venous thromboembolism prevention strategies in hospitalized patients: a systematic review
}

\author{
Subarna Thirugnanam', Ruxandra Pinto ${ }^{2}$, Deborah J Cook ${ }^{3}$, William H Geerts ${ }^{4}$ and Robert A Fowler ${ }^{3,4^{*}}$
}

\begin{abstract}
Introduction: Despite evidence-based guidelines for venous thromboembolism prevention, substantial variability is found in practice. Many economic evaluations of new drugs for thromboembolism prevention do not occur prospectively with efficacy studies and are sponsored by the manufacturers, raising the possibility of bias. We performed a systematic review of economic analyses of venous thromboembolism prevention in hospitalized patients to inform clinicians and policy makers about cost-effectiveness and the potential influence of sponsorship.

Methods: We searched MEDLINE, EMBASE, Cochrane Databases, ACP Journal Club, and Database of Abstracts of Reviews of Effects, from 1946 to September 2011. We extracted data on study characteristics, quality, costs, and efficacy.

Results: From 5,180 identified studies, 39 met eligibility and quality criteria. Each addressed pharmacologic prevention: low-molecular-weight heparins versus placebo (five), unfractionated heparin (12), warfarin (eight), one or another agents (five); fondaparinux versus enoxaparin (11); and rivaroxaban and dabigatran versus enoxaparin (two). Low-molecular-weight heparins were most economically attractive among most medical and surgical patients, whereas fondaparinux was favored for orthopedic patients. Fondaparinux was associated with increased bleeding events. Newer agents rivaroxaban and dabigatran may offer additional value. Of all economic evaluations, $64 \%$ were supported by manufacturers of a "new" agent. The new agent had a favorable outcome in 38 (97.4\%) of 39 evaluations [95\% confidence interval [CI] (86.5 to 99.9)]. Among studies supported by a pharmaceutical company, the sponsored medication was economically attractive in 24 (96.0\%) of 25 [95\% Cl, 80.0 to 99.9)]. We could not detect a consistent bias in outcome based on sponsorship; however, only a minority of studies were unsponsored.
\end{abstract}

Conclusion: Low-molecular-weight heparins and fondaparinux are the most economically attractive drugs for venous thromboembolism prevention in hospitalized patients. Approximately two thirds of evaluations were supported by the manufacturer of the new agent; such drugs were likely to be reported as economically favorable.

\section{Introduction}

Venous thromboembolism occurs in up to $40 \%$ of hospitalized medical and surgical patients in the absence of prophylactic anticoagulation [1,2]. Even with prophylaxis, the risk of venous thromboembolism in critically ill patients approaches $10 \%$ and has serious consequences: untreated pulmonary embolism has a mortality rate approaching 25\% [3-5]. Among critically ill patients,

\footnotetext{
* Correspondence: rob.fowler@sunnybrook.ca

${ }^{3}$ Department of Medicine, St. Joseph's Hospital and McMaster University,

Hamilton, Ontario, Canada

Full list of author information is available at the end of the article
}

those developing venous thromboembolism have longer intensive care unit and hospital stays, longer duration of mechanical ventilation, and higher hospital mortality [6]. Consequently, venous thromboembolism not only is associated with serious morbidity and mortality, but also has major implications for healthcare resource utilization.

Appropriate use of prophylaxis to prevent venous thromboembolism in patients at risk has been identified as one of the most important patient-safety interventions for hospitals [7]. However, substantial variability is found in the use of such prophylaxis in practice.

\section{Biomed Central}


Prevention is most commonly achieved with anticoagulant drugs. Because important decisions about pharmacologic interventions are made with knowledge of their economic consequences, formal economic analyses are useful tools to guide clinicians and policy makers about the value of drug interventions and their consequences $[8,9]$. However, many evaluations of new drugs do not occur prospectively with efficacy studies, and many are sponsored by the manufacturers, raising the possibility of bias.

We performed a systematic review of economic analyses of venous thromboembolism-prevention strategies in acutely ill hospitalized patients. Our objectives were to review and critically appraise the economic evaluations of a broad spectrum of strategies in diverse patient groups to help inform clinicians and policy makers about the cost-effectiveness of various approaches to venous thromboembolism prophylaxis.

\section{Materials and methods}

\section{Date sources and searches}

We searched MEDLINE, EMBASE, the Cochrane Database of Systematic Reviews, ACP Journal Club, Database of Abstracts of Reviews of Effects (DARE), and Cochrane Controlled Trials Register from 1946 to October 21, 2011, by using a combination of the following subject headings and text words: venous thrombosis, pulmonary embolism, low-molecular-weight heparin, $L M W H$, dalteparin, enoxaparin, nadroparin, tinzaparin, heparin, unfractionated heparin, UFH, anticoagulants, warfarin, aspirin, fondaparinux, rivaroxaban, dabigatran, intermittent pneumatic compression devices, compression stockings, vena cava filters, venous foot pump, economics, health care cost, cost-effectiveness analysis, cost-benefit analysis, and economic analysis (Additional file 1). No limits regarding publication type were initially applied. To identify additional potentially relevant studies, we checked the reference lists of identified systematic and narrative reviews and the personal files of the authors and collaborators. We also sent the full list of identified articles and inclusion criteria to venous thromboembolism experts in the field to identify additional published or relevant unpublished studies.

\section{Study selection}

From 5,180 potentially relevant citations, 4,816 were excluded based on title and abstract review (Figure 1). The full text versions of 89 manuscripts were retrieved for full evaluation. Two reviewers (ST, RF) independently assessed each of the articles and applied the following eligibility criteria: (a) the economic evaluation was based on data from randomized controlled trials or meta-analyses of randomized controlled trials; (b). the study described hospitalized patients; (c) the study compared at least two different venous thromboembolism prophylaxis strategies; (d) the study described drugacquisition costs, the costs of providing prophylaxis, costs of complications (including venous thromboembolism treatment and prophylaxis failures); and (e) the study described the effect of prophylaxis with respect to the number of venous thromboembolism events prevented and diagnosed. We excluded evaluations based on the following study designs: 1 . cohort studies or other observational studies; 2 . studies on outpatient use of venous thromboembolism prophylaxis; 3 . studies on the treatment of venous thromboembolism; 4 . studies examining the efficacy of short-term versus long-term venous thromboembolism prophylaxis; 5 . decision analytic models based on data from nonrandomized trials; 6 . studies examining anticoagulants for conditions other than venous thromboembolism, and seven letters, editorials, or narrative reviews of economic issues in venous thromboembolism prophylaxis. We also excluded studies appraised as low to moderate quality, as defined later.

\section{Data extraction and quality assessment}

We critically appraised each article by using established criteria [9]. Our goal was to include only those studies that adhered to a high methodologic quality. We assigned an ordinal score of quality based on the criteria set forth in the "User's guide to the medical literature: XIII. How to use an article on economic analysis of clinical practice" [9]. With a semiquantitative scale incorporating these characteristics, we assigned 1 point for each of the 12 categories. All studies were graded as high ( $\geq 9$ points), medium (5 to 8 points), or low (0 to 4 points) quality by two authors (ST, RF). Disagreements about the inclusion of individual studies were resolved by consensus between two authors (ST, RF). Of the 84 articles selected for full review, 50 were appraised as low or moderate quality, and the remaining 39 studies were selected for data abstraction.

We identified 10 economic evaluations of mechanical prophylaxis, including intermittent pneumatic compression devices, compression stockings, or vena cava filters [10-17]. None of these articles met our previously stated eligibility criteria.

From each included study, we abstracted the following: the patient group, venous thromboembolism prophylaxis strategy, duration of prophylaxis, time frame of the study, source of the outcome data, source of the cost data, incremental costs and benefits of each strategy, results of any sensitivity analyses, the country in which the study was performed, and the declared source of funding for the economic analysis. We attempted to contact authors of studies for which no external support was declared to ensure that this was the case. 

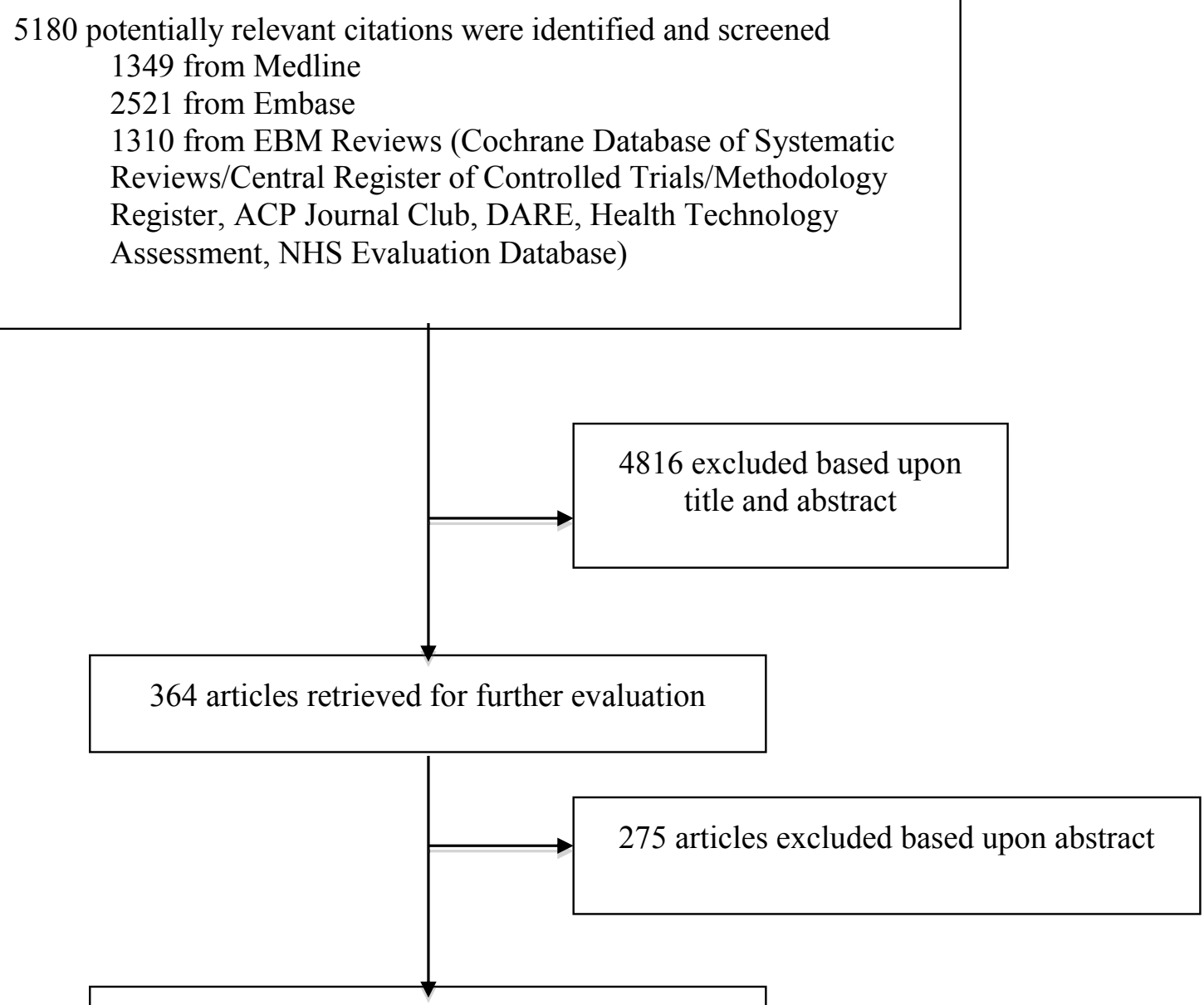

89 articles retrieved for evaluation of full text

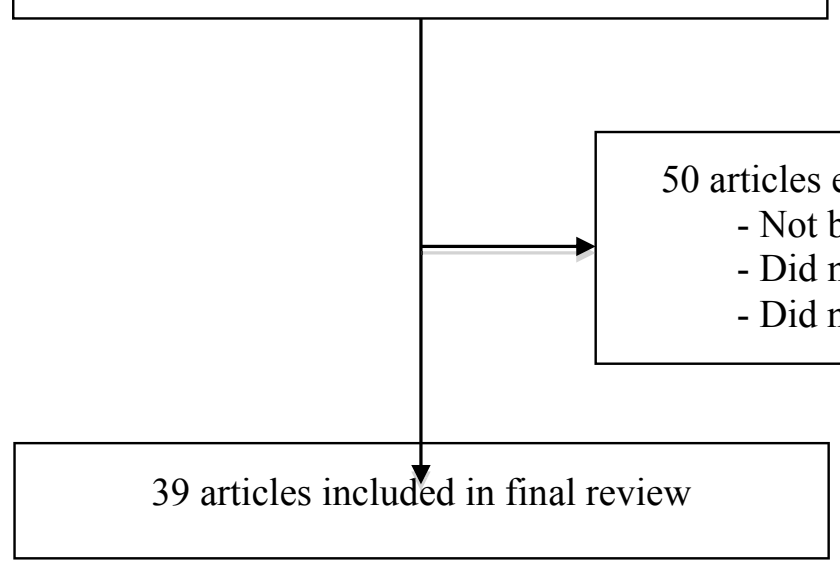

Figure 1 Study eligibility diagram

We abstracted the number of thrombotic events, costs, and complication rates of the prophylaxis and of the treatment of venous thromboembolism from each article. We then recorded or calculated the incremental cost-efficacy ratio for each venous thromboembolism prophylaxis strategy. If we were unable to calculate the incremental cost-efficacy ratio because of missing data, we attempted to contact the authors to obtain this ratio or original data. Costs were converted to 2009 US dollars and adjusted for country-specific temporal changes 
in gross domestic product [18-20]. We standardized the incremental effects we reported as "venous thromboembolism events avoided," "life-years or quality adjusted life-years gained' or 'deaths avoided per 1000 patients', as is commonly performed in venous thromboembolism literature. We chose in-hospital or near-term $(<90$ days) events for the primary comparisons whenever possible, as the short and longer-term effects of inpatient thromboprophylaxis are often greatest during this time period.

\section{Data synthesis and analysis}

Heterogeneity of the interventions, perspectives, and time-horizons precluded meta-analytic techniques to combine incremental cost-efficacy ratios into a single summary statistic. We summarized cost-effectiveness ratios by graphic representation of point estimates on a cost-efficacy plane. Categoric variables and proportions were compared by using the $\chi^{2}$ or Exact tests as appropriate.

\section{Results}

\section{Study comparisons, populations, and format}

Among the 39 studies included in this review, the following comparisons were made: low-molecular-weight heparins versus placebo (five) [20-24]; unfractionated heparin versus low-molecular-weight heparins (12) [21,23-33]; various low-molecular-weight heparins versus warfarin (eight) [34-41]; various low-molecularweight heparins compared with one another or other agents (five) [31,42-45]; fondaparinux versus enoxaparin (11) [46-56], rivaroxaban versus low-molecular-weight heparins or dabigatran [57], and dabigatran versus lowmolecular-weight heparin [58] (Tables 1 and 2).

Twenty-six evaluations were performed in orthopedic patients [25-27,34-43,46-58]; five in other surgical populations $[28,30-32,44]$, and eight in medical patients $[20-24,29,33,45]$. All 39 studies were either decision analytic models based on individual randomized controlled trials [20-22,24,26,28,31-49,51-58] or meta-analysis $[23,27,29,30,51]$.

\section{Study perspectives, time horizon, and funding}

The studies were conducted from a North American or European economic perspective: 18 of the studies were conducted in the United States [26,29,31-41,45,48, 51-53,55], six in the United Kingdom [21,23,25,46, $57,58]$, four in Canada $[23,28,34,51]$, and three in Italy $[27,30,44]$, two in Sweden $[42,47]$, and one each in Spain [43], Belgium [49], France [20], Norway [54], Switzerland [56], and Germany [24]. Seven studies were conducted from the perspective of the hospital $[24,41,42,44,45,49,53]$, four from a societal perspective $[20,28,30,32]$, nine from the perspective of another
Table 1 Study characteristics

\begin{tabular}{lc}
\hline Study characteristics & $\begin{array}{c}\text { Number of } \\
\text { studies }\end{array}$ \\
\hline $\begin{array}{l}\text { Thromboprophylaxis compared } \\
\text { Low-molecular-weight heparin versus placebo }\end{array}$ & 5 \\
$\quad$ Low-molecular-weight heparin versus & 12 \\
unfractionated heparin & \\
Low-molecular-weight heparin versus warfarin & 8 \\
Low-molecular-weight heparin versus & 11 \\
fondaparinux & \\
Other & 7 \\
Patient population & \\
$\quad$ Orthopedic surgery & 26 \\
Other surgical & 5 \\
Medical & 8 \\
Funding & \\
Industry & 25 \\
Other or unknown & 14 \\
Geographic perspective & \\
US & 18 \\
UK & 6 \\
Continental Europe & 11 \\
Canada & 4 \\
\hline
\end{tabular}

specific payer [23,31,33,40,54-58], and the remaining 19 studies did not clearly specify which perspective was used.

The prophylaxis time horizons considered were variable: either for 5 days $[33,51] ; 7$ days [25,28,34,38,44,46-50,53-56]; 6 to 14 days [23]; 10 days [31,32,37,40,41,52], 8 to 12 days [43]; 7 to 14 days [26]; 6 to 14 days [57], 10 to 14 days [20,22-24,27,30,35, $36,39,42-46$ ]; 30 days [29], 8 to 33 days [59], and another for 8 weeks [45]. Twenty-five studies received some sponsorship by pharmaceutical companies [20,22-25,28,30,33-38,40,41,43,44,46,47,50,54-56,58].

\section{Study quality}

All 39 studies that were of high quality were included in this systematic review (Table 2). Eighteen of these showed complete cost data but did not present complete effectiveness data [25-27,29,31,37-45,51,54-56]. The remaining 17 studies had complete cost and effectiveness data. Six of the studies obtained effectiveness data from meta-analysis $[23,25,27,31,50,57]$, whereas the remainder obtained effectiveness data from one or more randomized controlled trials. Six of these studies obtained effectiveness data from a single trial [20,22-24,33,41]. All studies, with the exception of one [23], obtained cost data from multiple sources, including actual and estimated healthcare system costs, randomized controlled trials, literature reviews, and other national government sources. All performed sensitivity analyses of some description. 
Table 2 Study description and quality assessment

\begin{tabular}{|c|c|c|c|c|c|c|}
\hline Article & Interventions compared & Patient group & $\begin{array}{l}\text { Were the } \\
\text { outcomes } \\
\text { accurately } \\
\text { measured? }\end{array}$ & $\begin{array}{l}\text { Were the } \\
\text { costs } \\
\text { accurately } \\
\text { measured? }\end{array}$ & $\begin{array}{l}\text { Was uncertainty } \\
\text { in analysis } \\
\text { determined? }\end{array}$ & $\begin{array}{l}\text { Were estimates and costs } \\
\text { related to the baseline } \\
\text { risk in treatment } \\
\text { population? }\end{array}$ \\
\hline $\begin{array}{l}\text { Pechevis, } \\
2000\end{array}$ & $\begin{array}{l}\text { Enoxaparin } 40 \mathrm{mg} \text { daily versus } \\
\text { placebo for 6-14 days }\end{array}$ & Medical & $\begin{array}{l}\text { Yes; } \\
\text { outcomes } \\
\text { taken from } \\
\text { RCTs }\end{array}$ & $\begin{array}{l}\text { Yes; data from } \\
\text { literature }\end{array}$ & Yes & Yes \\
\hline Lloyd, 2001 & $\begin{array}{l}\text { UFH 5,000 units twice daily versus } \\
\text { enoxaparin } 40 \text { mg daily for 6-14 } \\
\text { days }\end{array}$ & Medical & $\begin{array}{l}\text { Yes; } \\
\text { outcomes } \\
\text { taken from } \\
\text { RCTs }\end{array}$ & $\begin{array}{l}\text { Yes; data from } \\
\text { literature }\end{array}$ & Yes & Yes \\
\hline Lamy, 2002 & $\begin{array}{l}\text { Enoxaparin } 20 \mathrm{mg} \text { versus } 40 \mathrm{mg} \\
\text { versus placebo for } 6-14 \text { days }\end{array}$ & Medical & $\begin{array}{l}\text { Yes; } \\
\text { outcomes } \\
\text { taken from } \\
\text { RCTs }\end{array}$ & $\begin{array}{l}\text { Yes; data from } \\
\text { hospital, OHIP }\end{array}$ & Yes & Yes \\
\hline Offord, 2003 & $\begin{array}{l}\text { Enoxaparin } 40 \mathrm{mg} \text { daily versus } \\
\text { UFH 5,000 units twice daily versus } \\
\text { none for } 6-14 \text { days }\end{array}$ & Medical & $\begin{array}{l}\text { Yes; } \\
\text { outcomes } \\
\text { taken from } \\
\text { RCT/meta- } \\
\text { analysis }\end{array}$ & $\begin{array}{l}\text { Yes; data from } \\
\text { a hospital }\end{array}$ & Yes & Yes \\
\hline $\begin{array}{l}\text { Schadlich, } \\
2006\end{array}$ & $\begin{array}{l}\text { Enoxaparin } 40 \mathrm{mg} \text { versus UFH } \\
5,000 \text { units three times daily for } 6 \text { - } \\
14 \text { days }\end{array}$ & Medical & $\begin{array}{l}\text { Yes; } \\
\text { outcomes } \\
\text { taken from } \\
\text { RCTs/meta- } \\
\text { analysis }\end{array}$ & $\begin{array}{l}\text { Yes; data from } \\
\text { the German } \\
\text { Health System }\end{array}$ & Yes & Yes \\
\hline $\begin{array}{l}\text { Drummond, } \\
1994\end{array}$ & $\begin{array}{l}\text { UFH 5,000 units } 3 \text { times daily } \\
\text { versus enoxaparin } 40 \text { mg daily for } \\
7 \text { days }\end{array}$ & HFS & $\begin{array}{l}\text { Yes; } \\
\text { outcomes } \\
\text { taken from } \\
\text { RCTs }\end{array}$ & $\begin{array}{l}\text { Yes; data from } \\
\text { literature }\end{array}$ & Yes & Yes \\
\hline $\begin{array}{l}\text { Hawkins, } \\
1997\end{array}$ & $\begin{array}{l}\text { Enoxaparin } 30 \mathrm{mg} \text { daily versus } \\
\text { UFH 5,000 units for } 7 \text { days }\end{array}$ & THR & $\begin{array}{l}\text { Yes; } \\
\text { outcomes } \\
\text { taken from } \\
\text { RCTs }\end{array}$ & $\begin{array}{l}\text { Yes; data from } \\
\text { literature }\end{array}$ & Yes & Yes \\
\hline $\begin{array}{l}\text { Marchetti, } \\
1999\end{array}$ & $\begin{array}{l}\text { UFH } 5,000 \text { units twice daily versus } \\
\text { LMWH enoxaparin } 40 \text { mg daily } \\
\text { for } 14 \text { days }\end{array}$ & THR & $\begin{array}{l}\text { Yes; } \\
\text { outcomes } \\
\text { taken from } \\
\text { RCTs/meta- } \\
\text { analysis }\end{array}$ & $\begin{array}{l}\text { Yes; data from } \\
\text { literature }\end{array}$ & Yes & Yes \\
\hline $\begin{array}{l}\text { McGarry, } \\
2004\end{array}$ & $\begin{array}{l}\text { UFH 5,000 units twice daily versus } \\
\text { enoxaparin } 40 \text { mg daily versus } \\
\text { nothing for } 30 \text { days }\end{array}$ & Medical & $\begin{array}{l}\text { Yes; } \\
\text { outcomes } \\
\text { taken from } \\
\text { RCTs/meta- } \\
\text { analysis }\end{array}$ & $\begin{array}{l}\text { Yes; data from } \\
\text { literature }\end{array}$ & Yes & Yes \\
\hline $\begin{array}{l}\text { Deitelzweig, } \\
2008\end{array}$ & $\begin{array}{l}\text { UFH 5,000 units twice daily versus } \\
\text { enoxaparin } 40 \text { mg daily versus } \\
\text { nothing for } 5 \text { days }\end{array}$ & Medical & $\begin{array}{l}\text { Yes; } \\
\text { outcomes } \\
\text { taken from } \\
\text { RCTs }\end{array}$ & $\begin{array}{l}\text { Yes; data from } \\
\text { literature }\end{array}$ & Yes & Yes \\
\hline Wade, 2008 & $\begin{array}{l}\text { UFH 5,000 units } 3 \text { times daily } \\
\text { versus dalteparin 5,000 units daily } \\
\text { for } 10 \text { days }\end{array}$ & $\begin{array}{l}\text { Gynecology } \\
\text { oncology } \\
\text { surgery }\end{array}$ & $\begin{array}{l}\text { Yes; } \\
\text { outcomes } \\
\text { taken from } \\
\text { RCTs }\end{array}$ & $\begin{array}{l}\text { Yes; data from } \\
\text { literature }\end{array}$ & Yes & Yes \\
\hline Lloyd, 1997 & $\begin{array}{l}\text { UFH } 5,000 \text { units twice daily/3 } \\
\text { times daily versus nadroparin for } \\
10-14 \text { days }\end{array}$ & $\begin{array}{l}\text { Orthopedic and } \\
\text { general surgery }\end{array}$ & $\begin{array}{l}\text { Yes; } \\
\text { outcomes } \\
\text { taken from } \\
\text { meta- } \\
\text { analysis }\end{array}$ & $\begin{array}{l}\text { Yes; data from } \\
\text { published rates } \\
\text { of pay, costs } \\
\text { from a hospital }\end{array}$ & Yes & Yes \\
\hline $\begin{array}{l}\text { Heerey, } \\
2005\end{array}$ & $\begin{array}{l}\text { Dalteparin } 2,500 \text { units versus } \\
5,000 \text { units versus UFH for } 10 \\
\text { days }\end{array}$ & $\begin{array}{l}\text { Abdominal } \\
\text { surgery }\end{array}$ & $\begin{array}{l}\text { Yes; } \\
\text { outcomes } \\
\text { taken from } \\
\text { RCTs }\end{array}$ & $\begin{array}{l}\text { Yes; data from } \\
\text { Medicare } \\
\text { reimbursement }\end{array}$ & Yes & Yes \\
\hline $\begin{array}{l}\text { O'Brien, } \\
1994\end{array}$ & $\begin{array}{l}\text { Enoxaparin } 30 \mathrm{mg} \text { twice daily } \\
\text { versus warfarin for } 7 \text { days }\end{array}$ & THR & $\begin{array}{l}\text { Yes; } \\
\text { outcomes } \\
\text { taken from } \\
\text { RCTs }\end{array}$ & $\begin{array}{l}\text { Yes; data from } \\
\text { literature }\end{array}$ & Yes & Yes \\
\hline
\end{tabular}


Table 2 Study description and quality assessment (Continued)

\begin{tabular}{|c|c|c|c|c|c|c|}
\hline $\begin{array}{l}\text { Menzin, } \\
1995\end{array}$ & $\begin{array}{l}\text { Enoxaparin } 30 \mathrm{mg} \text { twice daily } \\
\text { versus warfarin (INR 2-3) versus } \\
\text { nothing for 5-14 days }\end{array}$ & THR & $\begin{array}{l}\text { Yes; } \\
\text { outcomes } \\
\text { taken from } \\
\text { RCTs }\end{array}$ & $\begin{array}{l}\text { Yes; data from } \\
\text { literature }\end{array}$ & Yes & Yes \\
\hline Hull, 1997 & $\begin{array}{l}\text { Warfarin versus tinzaparin } 175 \\
\text { units/kg for } 14 \text { days }\end{array}$ & THR, TKR & $\begin{array}{l}\text { Yes; } \\
\text { outcomes } \\
\text { taken from } \\
\text { RCTs }\end{array}$ & $\begin{array}{l}\text { Yes; data from } \\
\text { literature }\end{array}$ & Yes & Yes \\
\hline $\begin{array}{l}\text { Hawkins, } \\
1998\end{array}$ & $\begin{array}{l}\text { Enoxaparin } 30 \mathrm{mg} \text { twice daily } \\
\text { versus warfarin for } 10 \text { days }\end{array}$ & TKR & $\begin{array}{l}\text { Yes; } \\
\text { outcomes } \\
\text { taken from } \\
\text { RCTs }\end{array}$ & $\begin{array}{l}\text { Yes; data from } \\
\text { literature }\end{array}$ & Yes & Yes \\
\hline Francis, 1999 & $\begin{array}{l}\text { Dalteparin } 2,500 \text { units, then } 5,000 \\
\text { units versus warfarin for } 10 \text { days }\end{array}$ & THR & $\begin{array}{l}\text { Yes; } \\
\text { outcomes } \\
\text { taken from } \\
\text { RCTs }\end{array}$ & $\begin{array}{l}\text { Yes; costs from } \\
\text { participating } \\
\text { hospitals in RCT }\end{array}$ & Yes; for costs & Yes \\
\hline $\begin{array}{l}\text { Botteman, } \\
2002\end{array}$ & $\begin{array}{l}\text { Enoxaparin } 30 \mathrm{mg} \text { daily versus } \\
\text { warfarin } 5 \mathrm{mg} \text { daily for } 7 \text { days }\end{array}$ & THR & $\begin{array}{l}\text { Yes; } \\
\text { outcomes } \\
\text { taken from } \\
\text { RCTs }\end{array}$ & $\begin{array}{l}\text { Yes; data from } \\
\text { literature }\end{array}$ & Yes & Yes \\
\hline $\begin{array}{l}\text { Caprini, } \\
2002\end{array}$ & $\begin{array}{l}\text { Enoxaparin } 30 \text { mg twice daily for } \\
7 \text { days versus UFH } 5,000 \text { units } 3 \\
\text { times daily and warfarin for } 10 \\
\text { days }\end{array}$ & THR & $\begin{array}{l}\text { Yes; } \\
\text { outcomes } \\
\text { taken from } \\
\text { RCTs }\end{array}$ & $\begin{array}{l}\text { Yes; data from } \\
\text { literature }\end{array}$ & Yes & Yes \\
\hline Levin, 2001 & $\begin{array}{l}\text { Desirudin } 15 \text { mg twice daily } \\
\text { versus enoxaparin } 40 \text { mg daily for } \\
10 \text { days }\end{array}$ & THR & $\begin{array}{l}\text { Yes; } \\
\text { outcomes } \\
\text { taken from } \\
\text { RCTs }\end{array}$ & $\begin{array}{l}\text { Yes; data from } \\
\text { literature }\end{array}$ & Yes & Yes \\
\hline $\begin{array}{l}\text { Honorato, } \\
2004\end{array}$ & $\begin{array}{l}\text { Bemiparin } 3,500 \text { units daily versus } \\
\text { enoxaparin } 40 \text { mg daily for 8-12 } \\
\text { days }\end{array}$ & TKR & $\begin{array}{l}\text { Yes; } \\
\text { outcomes } \\
\text { taken from } \\
\text { RCTs }\end{array}$ & $\begin{array}{l}\text { Yes; data from } \\
\text { National Health } \\
\text { Care Institute, } \\
\text { pharmacists } \\
\text { association }\end{array}$ & Yes & Yes \\
\hline $\begin{array}{l}\text { Attanasio, } \\
2001\end{array}$ & $\begin{array}{l}\text { Dermatan sulfate } 300 \text { mg daily } \\
\text { versus UFH } 5,000 \text { units } 3 \text { times } \\
\text { daily for } 7 \text { days }\end{array}$ & $\begin{array}{l}\text { Surgical } \\
\text { oncology }\end{array}$ & $\begin{array}{l}\text { Yes; } \\
\text { outcomes } \\
\text { taken from } \\
\text { RCTs }\end{array}$ & $\begin{array}{l}\text { Yes - data from } \\
\text { hospital costs }\end{array}$ & Yes & Yes \\
\hline \multirow[t]{2}{*}{ Wade, 2001} & $\begin{array}{l}\text { Tinzaparin } 3,500 \text { units versus } \\
\text { enoxaparin } 30 \text { mg twice daily for } \\
8 \text { weeks }\end{array}$ & $\begin{array}{l}\text { Spinal cord } \\
\text { injury }\end{array}$ & $\begin{array}{l}\text { Yes; } \\
\text { outcomes } \\
\text { taken from } \\
\text { RCTs }\end{array}$ & $\begin{array}{l}\text { Yes; data from } \\
\text { different } \\
\text { hospitals, DRG }\end{array}$ & Yes & Yes \\
\hline & & & & & & $\begin{array}{l}\text { Were estimates and costs } \\
\text { related to the baseline } \\
\text { risk in treatment } \\
\text { population- are these } \\
\text { results generalizable? }\end{array}$ \\
\hline $\begin{array}{l}\text { Gordois, } \\
2003\end{array}$ & $\begin{array}{l}\text { Enoxaparin } 40 \text { mg daily versus } \\
\text { fondaparinux } 2.5 \text { mg daily for } 5-9 \\
\text { days }\end{array}$ & THR, HFS & $\begin{array}{l}\text { Yes; } \\
\text { outcomes } \\
\text { taken from } \\
\text { RCTs }\end{array}$ & $\begin{array}{l}\text { Yes; data from } \\
\text { NICE }\end{array}$ & Yes & Yes \\
\hline $\begin{array}{l}\text { Lundkvist, } \\
2003\end{array}$ & $\begin{array}{l}\text { Fondaparinux } 2.5 \mathrm{mg} \text { daily versus } \\
\text { enoxaparin } 40 \mathrm{mg} \text { daily for } 7 \text { days }\end{array}$ & THR, HFS & $\begin{array}{l}\text { Yes; } \\
\text { outcomes } \\
\text { taken from } \\
\text { RCTs }\end{array}$ & $\begin{array}{l}\text { Yes; data from } \\
\text { literature }\end{array}$ & Yes & Yes \\
\hline Wade, 2003 & $\begin{array}{l}\text { Fondaparinux } 2.5 \mathrm{mg} \text { daily versus } \\
\text { enoxaparin } 40 \text { mg daily versus } 30 \\
\text { mg twice daily for } 7-10 \text { days }\end{array}$ & HFS & $\begin{array}{l}\text { Yes; } \\
\text { outcomes } \\
\text { taken from } \\
\text { RCTs }\end{array}$ & $\begin{array}{l}\text { Yes; data from } \\
\text { literature }\end{array}$ & Yes & Yes \\
\hline Szucs, 2003 & $\begin{array}{l}\text { Fondaparinux } 2.5 \mathrm{mg} \text { daily versus } \\
\text { enoxaparin } 40 \mathrm{mg} \text { daily for } 7 \text { days }\end{array}$ & THR, TKR HFS & $\begin{array}{l}\text { Yes; } \\
\text { outcomes } \\
\text { taken from } \\
\text { RCTs }\end{array}$ & $\begin{array}{l}\text { Yes; data from } \\
\text { literature and } \\
\text { surveys in } \\
\text { Switzerland }\end{array}$ & Yes & Yes \\
\hline
\end{tabular}


Table 2 Study description and quality assessment (Continued)

\begin{tabular}{|c|c|c|c|c|c|c|}
\hline $\begin{array}{l}\text { Sullivan, } \\
2004\end{array}$ & $\begin{array}{l}\text { Fondaparinux } 2.5 \mathrm{mg} \text { daily versus } \\
\text { enoxaparin } 40 \mathrm{mg} \text { daily for } 7 \text { days }\end{array}$ & THR, TKR HFS & $\begin{array}{l}\text { Yes; } \\
\text { outcomes } \\
\text { taken from } \\
\text { RCTs }\end{array}$ & $\begin{array}{l}\text { Yes; costs from } \\
\text { review of } 220 \\
\text { acute care } \\
\text { hospitals }\end{array}$ & Yes & Yes \\
\hline $\begin{array}{l}\text { Dranitsaris, } \\
2004\end{array}$ & $\begin{array}{l}\text { Fondaparinux } 2.5 \mathrm{mg} \text { daily versus } \\
\text { enoxaparin } 40 \mathrm{mg} \text { daily for } 7 \text { days }\end{array}$ & THR, HFS & $\begin{array}{l}\text { Yes; } \\
\text { outcomes } \\
\text { taken from a } \\
\text { meta- } \\
\text { analysis }\end{array}$ & $\begin{array}{l}\text { Data from } \mathrm{ClHI}, \\
\text { surveys }\end{array}$ & Yes & Yes \\
\hline Spruill, 2004 & $\begin{array}{l}\text { Fondaparinux } 2.5 \mathrm{mg} \text { daily versus } \\
\text { enoxaparin } 30 \mathrm{mg} \text { twice daily for } \\
4-5 \text { days }\end{array}$ & TKA & $\begin{array}{l}\text { Yes; } \\
\text { outcomes } \\
\text { taken from } \\
\text { RCTs }\end{array}$ & $\begin{array}{l}\text { Yes; data from } \\
\text { literature }\end{array}$ & Yes & Yes \\
\hline Spruill, 2004 & $\begin{array}{l}\text { Fondaparinux } 2.5 \mathrm{mg} \text { daily versus } \\
\text { enoxaparin } 30 \mathrm{mg} \text { twice daily for } \\
10 \text { days }\end{array}$ & THR & $\begin{array}{l}\text { Yes; } \\
\text { outcomes } \\
\text { taken from } \\
\text { RCTs }\end{array}$ & $\begin{array}{l}\text { Yes; data from } \\
\text { literature }\end{array}$ & Yes & Yes \\
\hline Wade, 2004 & $\begin{array}{l}\text { Fondaparinux } 2.5 \mathrm{mg} \text { daily versus } \\
\text { enoxaparin } 40 \mathrm{mg} \text { daily for } 7 \text { days }\end{array}$ & HFS & $\begin{array}{l}\text { Yes; } \\
\text { outcomes } \\
\text { taken from } \\
\text { RCTs }\end{array}$ & $\begin{array}{l}\text { Yes; data from } \\
\text { literature }\end{array}$ & Yes & Yes \\
\hline $\begin{array}{l}\text { Bjorvatn, } \\
2005\end{array}$ & $\begin{array}{l}\text { Fondaparinux } 2.5 \mathrm{mg} \text { daily versus } \\
\text { enoxaparin } 40 \mathrm{mg} \text { daily for } 7 \text { days }\end{array}$ & THR, TKR HFS & $\begin{array}{l}\text { Yes; } \\
\text { outcomes } \\
\text { taken from } \\
\text { RCTs }\end{array}$ & $\begin{array}{l}\text { Yes; data from } \\
\text { Norwegian } \\
\text { national } \\
\text { sources }\end{array}$ & Yes & Yes \\
\hline $\begin{array}{l}\text { Wolowacz } \\
2009\end{array}$ & $\begin{array}{l}\text { THR Dabigatran } 220 \text { mg daily } \\
\text { versus enoxaparin } 40 \text { mg daily for } \\
28-35 \text { days TKR Dabigatran } 220 \\
\text { mg daily for versus enoxaparin } 40 \\
\text { mg daily 6-10 days }\end{array}$ & THR, TKR & $\begin{array}{l}\text { Yes; } \\
\text { outcomes } \\
\text { taken from } \\
\text { RCTs }\end{array}$ & $\begin{array}{l}\text { Yes; data from } \\
\text { UK national } \\
\text { sources }\end{array}$ & Yes & Yes \\
\hline $\begin{array}{l}\text { McCullagh, } \\
2009\end{array}$ & $\begin{array}{l}\text { THR Dabigatran } 220 \text { mg daily for } \\
35 \text { days versus rivaroxaban } 10 \text { mg } \\
\text { daily for } 35 \text { days versus } \\
\text { enoxaparin } 40 \text { mg daily for } 14 \\
\text { days TKR Dabigatran } 220 \text { mg daily } \\
\text { for } 14 \text { days versus rivaroxaban } 10 \\
\text { mg daily for } 10 \text { days versus } \\
\text { enoxaparin } 40 \text { mg daily for } 10 \\
\text { days }\end{array}$ & THR, TKR & $\begin{array}{l}\text { Yes; } \\
\text { outcomes } \\
\text { taken from } \\
\text { RCTs }\end{array}$ & $\begin{array}{l}\text { Yes; data from } \\
\text { literature and } \\
\text { Irish national } \\
\text { sources }\end{array}$ & Yes & Yes \\
\hline $\begin{array}{l}\text { Pechevis, } \\
2000\end{array}$ & Yes & $N / R$ & No & Yes & Yes & Yes \\
\hline Lloyd, 2001 & Yes & $N / R$ & No & Yes & Yes & Yes \\
\hline Lamy, 2002 & Yes & $N / R$ & No & Yes & Yes & Yes \\
\hline Offord, 2003 & Yes & $N / R$ & No & Yes & Yes & Yes \\
\hline $\begin{array}{l}\text { Schadlich, } \\
2006\end{array}$ & Incompletely & $N / R$ & No & Yes & Yes & Yes \\
\hline $\begin{array}{l}\text { Drummond, } \\
1994\end{array}$ & Incompletely & $N / R$ & No & Yes & Yes & Likely \\
\hline $\begin{array}{l}\text { Hawkins, } \\
1997\end{array}$ & Yes & $N / R$ & No & Yes & Yes & Yes \\
\hline $\begin{array}{l}\text { Marchetti, } \\
1999\end{array}$ & Incompletely & $N / R$ & No & Yes & Yes & Yes \\
\hline $\begin{array}{l}\text { Etchells, } \\
1999\end{array}$ & Yes & $N / R$ & No & Yes & Yes & Yes \\
\hline $\begin{array}{l}\text { McGarry, } \\
2004\end{array}$ & Incompletely & $N / R$ & No & Yes & Yes & Yes \\
\hline Article & $\begin{array}{l}\text { Were incremental costs and } \\
\text { outcomes measured? }\end{array}$ & $\begin{array}{l}\text { Do } \\
\text { incremental } \\
\text { costs and } \\
\text { outcomes } \\
\text { differ between } \\
\text { subgroups? }\end{array}$ & $\begin{array}{l}\text { Does } \\
\text { allowance } \\
\text { for } \\
\text { uncertainty } \\
\text { change } \\
\text { results? }\end{array}$ & $\begin{array}{l}\text { Are } \\
\text { prophylaxis } \\
\text { benefits worth } \\
\text { the harm and } \\
\text { costs? }\end{array}$ & $\begin{array}{l}\text { Generalizability: } \\
\text { could other } \\
\text { patient } \\
\text { populations } \\
\text { expect similar } \\
\text { outcomes? }\end{array}$ & $\begin{array}{l}\text { Generalizability: could } \\
\text { other patient } \\
\text { populations expect to } \\
\text { experience similar costs? }\end{array}$ \\
\hline
\end{tabular}


Table 2 Study description and quality assessment (Continued)

\begin{tabular}{|c|c|c|c|c|c|c|}
\hline $\begin{array}{l}\text { Heerey, } \\
2005\end{array}$ & Incompletely & $N / R$ & No & Yes & Yes & Likely \\
\hline $\begin{array}{l}\text { Deitelzweig, } \\
2008\end{array}$ & Yes & $N / R$ & No & Yes & Yes & Likely \\
\hline Wade, 2008 & Yes & Yes & Yes & Yes & Yes & Yes \\
\hline $\begin{array}{l}\text { O'Brien, } \\
1994\end{array}$ & Yes & $N / R$ & No & Yes & Yes & Yes \\
\hline $\begin{array}{l}\text { Menzin, } \\
1995\end{array}$ & Yes & $N / R$ & No & Yes & Yes & Yes \\
\hline Hull, 1997 & Yes & $N / R$ & No & Yes & Yes & Yes \\
\hline $\begin{array}{l}\text { Hawkins, } \\
1998\end{array}$ & Yes & $N / R$ & No & Yes & Yes & Yes \\
\hline Francis, 1999 & Yes & Yes & Yes & Likely & Yes & Yes \\
\hline $\begin{array}{l}\text { Botteman, } \\
2002\end{array}$ & Incompletely & $N / R$ & No & Yes & Perhaps & Yes \\
\hline $\begin{array}{l}\text { Nerurkar, } \\
2002\end{array}$ & Incompletely & $N / R$ & No & Yes & Perhaps & Yes \\
\hline Levin, 2001 & Incompletely & $N / R$ & No & Yes & Yes & Likely \\
\hline \multirow{2}{*}{$\begin{array}{l}\text { Caprini, } \\
2002\end{array}$} & Yes & Yes & No & Yes & Yes & Likely \\
\hline & $\begin{array}{l}\text { Were incremental costs and } \\
\text { outcomes measured? }\end{array}$ & $\begin{array}{l}\text { Do } \\
\text { incremental } \\
\text { costs and } \\
\text { outcomes } \\
\text { differ between } \\
\text { subgroups? }\end{array}$ & $\begin{array}{l}\text { Does } \\
\text { allowance } \\
\text { for } \\
\text { uncertainty } \\
\text { change } \\
\text { results? }\end{array}$ & $\begin{array}{l}\text { Are } \\
\text { prophylaxis } \\
\text { benefits worth } \\
\text { the harm and } \\
\text { costs? }\end{array}$ & $\begin{array}{l}\text { Generalizability: } \\
\text { could other } \\
\text { patient } \\
\text { populations } \\
\text { expect similar } \\
\text { outcomes? }\end{array}$ & $\begin{array}{l}\text { Generalizability: could } \\
\text { other patient } \\
\text { populations expect to } \\
\text { experience similar costs? }\end{array}$ \\
\hline $\begin{array}{l}\text { Honorato, } \\
2004\end{array}$ & Yes & $N / R$ & No & Yes & Yes & Yes \\
\hline Wade, 2001 & Incompletely & $N / R$ & No & Yes & Yes & Yes \\
\hline $\begin{array}{l}\text { Gordois, } \\
2003\end{array}$ & Yes & $N / R$ & No & Yes & Yes & Yes \\
\hline Wade, 2003 & Yes & $N / R$ & No & Yes & Yes & Yes \\
\hline $\begin{array}{l}\text { Annemans, } \\
2004\end{array}$ & Yes & $N / R$ & No & Yes & Yes & Yes \\
\hline $\begin{array}{l}\text { Attanasio, } \\
2001\end{array}$ & Yes & $N / R$ & No & Yes & Yes & Yes \\
\hline Szucs, 2003 & Yes & Yes & No & Yes & Yes & Yes \\
\hline $\begin{array}{l}\text { Sullivan, } \\
2004\end{array}$ & Yes & Yes & No & Yes & Yes & Yes \\
\hline $\begin{array}{l}\text { Dranitsaris, } \\
2004\end{array}$ & Yes & $N / R$ & No & Yes & Yes & Yes \\
\hline Spruill, 2004 & Yes & $N / R$ & No & Yes & Yes & Yes \\
\hline Spruill, 2004 & Yes & $N / R$ & No & Yes & Yes & Yes \\
\hline Wade, 2004 & Yes & $N / R$ & No & Yes & Yes & Yes \\
\hline $\begin{array}{l}\text { Bjorvatn, } \\
2005\end{array}$ & Yes & Yes & No & Yes & Yes & Yes \\
\hline $\begin{array}{l}\text { Wolowacz } \\
2009\end{array}$ & Yes & Yes & No & Yes & Yes & Yes \\
\hline $\begin{array}{l}\text { McCullagh } \\
2009\end{array}$ & Yes & Yes & No & Yes & Yes & Yes \\
\hline
\end{tabular}

CIHI, Canadian Institute for Health Information; DRG, diagnosis-related group; HFS, hip fracture surgery; NICE, National Centre for Clinical Excellence $\mathrm{N} / \mathrm{R}$, not reported; OHIP, Ontario Health Insurance Plan; RCT, randomized controlled trial; THR, total hip replacement; TKR, total knee replacement; UFH, unfractionated heparin.

\section{Cost and effect estimates}

\section{Low Molecular Weight Heparins versus Placebo}

Among the five studies comparing low-molecular-weight heparins with placebo in medical patients, enoxaparin was the most economically attractive strategy in all five studies and dominant in two [20,22], with incremental cost-efficacy ratios ranging from $\$ 83$ to $\$ 1,711$ per venous thromboembolism event avoided in three others 
$[21,23,24]$; cost per life-year or quality-adjusted life-year gained were not investigated (Table 3 ). Sensitivity analysis did not alter these findings. Four of the five studies were sponsored by the manufacturer of enoxaparin [20-22,24].

\section{Unfractionated Heparin versus Low Molecular Weight Heparins}

Among the 12 studies comparing low-molecular-weight heparins with unfractionated heparin among medical and surgical patients, 11 found that low-molecularweight heparins were more effective (Table 3 and Figure $2)$. Eight of the 12 studies comparing low-molecularweight heparins with unfractionated heparin found lowmolecular-weight heparins to be the dominant strategy $[21,23-25,27,29,30,32,33]$. Two studies reported an incremental cost-efficacy ratio of $\$ 1,180$ and $\$ 1,445$ per venous thromboembolism event avoided when using enoxaparin [26,29], and two studies found incremental cost-effectiveness ratios of $\$ 10,360$ per death avoided and $\$ 20,337$ per quality-adjusted life-year gained with low-molecular-weight heparins. A single study of venous thromboembolism prevention among patients undergoing colorectal cancer surgery found no difference in efficacy, yet costs of low-molecular-weight heparins were greater [28]. Three studies reported increased bleeding risk with low-molecular-weight heparins, and three studies reported lower risk. Sensitivity analyses did not change these results. Of these 12 studies, eight received financial support from the manufacturer of the low-molecular-weight heparins [23-26,28-30,33].

\section{Warfarin versus Low-Molecular-Weight Heparins}

Low-molecular-weight heparins were reported to be economically more attractive than warfarin in all eight studies among surgical patients, with incremental costefficacy ratios of $\$ 874$ to $\$ 26,711$ per venous thromboembolism event avoided in five of the comparisons $[34,35,37,38,40]$, the dominant strategy in three comparisons $[36,39,41]$. Long-term outcomes varied widely, with $\$ 16,200$ to $\$ 334,055$ per death avoided, $\$ 32,158$ per life-year and $\$ 4,340$ per quality-adjusted life-year gained (Table 3 and Figure 3) [34,38]. Sensitivity analyses did not change the results in individual studies. Of these eight studies, seven received pharmaceutical sponsorship [34-38,40,41].

\section{Low-Molecular-Weight Heparins versus One another, and} Other Comparisons

Within the studies comparing low-molecular-weight heparins with one another and with other anticoagulants among surgical patients, bemiparin and dermatan sulfate were the dominant prophylaxis over enoxaparin [43,44]. Desirudin had an incremental cost-effectiveness ratio of $\$ 3,794$ per life-year gained, whereas enoxaparin was favored over tinzaparin but was more expensive (Table 3) [45]. Dalteparin, 5,000 units once daily, was more efficacious than dalteparin, 2,500 units, with an incremental cost-effectiveness ratio of $\$ 24,357$ per qualityadjusted life-year gained [30].

Fondaparinux versus Low Molecular Weight Heparins

Among the 11 studies comparing fondaparinux with enoxaparin, all were conducted in orthopedic surgery patients, and all concluded that fondaparinux was economically attractive. In six, fondaparinux was dominant [47,51-53,55,56], and in one, enoxaparin [48] (Table 3 and Figure 4). In four studies, incremental cost-effectiveness ratios of fondaparinux over enoxaparin were $\$ 158$ to $\$ 1,077$ per venous thromboembolism event avoided, $\$ 104$ to $\$ 6,782$ per death avoided, and $\$ 32,144$ per quality-adjusted life-year gained [46,49,53,54]. In eight of 11 studies, fondaparinux was associated with increased bleeding risk. Sensitivity analyses of the various costs did not alter the findings. The manufacturer of fondaparinux provided sponsorship for six of the 11 studies.

Dabigatran and Rivaroxaban versus Low Molecular Weight Heparins

Among orthopedic patients, dabigatran, in comparison with enoxaparin, was dominant, with cost savings of $\$ 103,050$ and $\$ 8,162$ and six and seven quality-adjusted life-years gained per 1,000 patients with a total hip replacement and total knee replacement, respectively [58]. Comparing rivaroxaban, dabigatran, and enoxaparin among orthopedic surgery patients, rivaroxaban was dominant, with cost savings of $\$ 24,104$ and $\$ 213,452$ and 7 lifeyears gained per 1,000 patients with a total hip replacement and total knee replacement, respectively [57].

\section{Sponsorship and Economic Comparisons}

When comparing different populations and the different modes of venous thromboembolism prophylaxis, we observed several interesting trends. All studies comparing fondaparinux, rivaroxaban, or dabigatran with enoxaparin were performed in orthopedic patients, and the remainder of the studies in this patient population examined various low-molecular-weight heparins or warfarin. Sixteen of the 25 studies among orthopedic patients were sponsored in some manner by the pharmaceutical industry: six studies favored fondaparinux [46,47,50,54-56]; one, rivaroxaban [57]; one, dabigatran [58]; and the remainder favored low-molecular-weight heparins [25,34-38,40,41,43]. In comparison, five of the eight studies conducted in medical patients compared enoxaparin with placebo [20-24]; two compared unfractionated heparin with enoxaparin [29,33]; and the final one compared enoxaparin with tinzaparin [45]. Five of these eight studies were sponsored by pharmaceutical companies, and all studies favored enoxaparin $[20,22-24]$. Of the five studies in other surgical populations, three were sponsored by the pharmaceutical industry, and these studies favored unfractionated heparin [28], nadroparin [30], and dermatan sulfate [44]. 
Table 3 Incremental costs, effects, and cost-efficacy ratios for the different modes of venous thromboembolism prophylaxis

\begin{tabular}{|c|c|c|c|c|c|c|}
\hline \multicolumn{7}{|c|}{ Low-molecular-weight heparins versus placebo } \\
\hline Reference & $\begin{array}{l}\text { Patient } \\
\text { population }\end{array}$ & $\begin{array}{l}\text { Incremental } \\
\text { cost (USD) }\end{array}$ & $\begin{array}{l}\text { Incremental effects } \\
\text { (VTE avoided or life- } \\
\text { years or QALYS } \\
\text { gained) }\end{array}$ & $\begin{array}{l}\text { ICER (USD/VTE event } \\
\text { avoided or life-years } \\
\text { or QALYS gained) }\end{array}$ & Bleeding complications & $\begin{array}{l}\text { Most } \\
\text { economically } \\
\text { attractive } \\
\text { drug }\end{array}$ \\
\hline *Pechevis, 2000 & Medical & $\begin{array}{l}\text { Net saving } \\
\text { (value not } \\
\text { reported) per } \\
1,000 \text { patients } \\
\text { with enoxaparin }\end{array}$ & $\begin{array}{l}94 \text { DVT/PE avoided, } \\
\text { four lives (estimated } 12 \\
\text { life-years) gained, per } \\
\text { 1,000 patients with } \\
\text { enoxaparin }\end{array}$ & Enoxaparin dominant & Not reported & Enoxaparin \\
\hline Lloyd, 2001 & Medical & $\begin{array}{l}\$ 20,680 \text { per } \\
1,000 \text { patients } \\
\text { with enoxaparin }\end{array}$ & $\begin{array}{l}20 \text { VTE events avoided } \\
\text { per } 1,000 \text { patients with } \\
\text { enoxaparin }\end{array}$ & $\begin{array}{l}\$ 1,034 \text { per VTE } \\
\text { avoided with } \\
\text { enoxaparin }\end{array}$ & $\begin{array}{l}\text { Six more major bleeding } \\
\text { events per } 1,000 \text { patients with } \\
\text { enoxaparin }\end{array}$ & Enoxaparin \\
\hline *Lamy, 2002 & Medical & $\begin{array}{l}\$ 1,910 \text { per } \\
1,000 \text { patients } \\
\text { in tertiary care } \\
\text { setting with } \\
\text { enoxaparin }\end{array}$ & $\begin{array}{l}\text { 2.3\% fewer VTE events } \\
\text { with enoxaparin }\end{array}$ & $\begin{array}{l}\$ 83 \text { per VTE avoided } \\
\text { with enoxaparin }\end{array}$ & Not reported & Enoxaparin \\
\hline *Offord, 2004 & Medical & $\begin{array}{l}\text { Net saving } \\
(\$ 26,478) \text { per } \\
1,000 \text { patients } \\
\text { with enoxaparin }\end{array}$ & $\begin{array}{l}14 \text { VTE events and } 3.5 \\
\text { deaths avoided per } \\
\text { 1,000 patients with } \\
\text { enoxaparin }\end{array}$ & Enoxaparin dominant & Not reported & Enoxaparin \\
\hline *Schaldich, 2006 & Medical & $\begin{array}{l}\$ 44,665 \text { per } \\
1,000 \text { patients } \\
\text { with enoxaparin }\end{array}$ & $\begin{array}{l}26 \text { VTE events avoided } \\
\text { per 1,000 patients with } \\
\text { enoxaparin }\end{array}$ & $\begin{array}{l}\text { \$1, } 711 \text { per VTE } \\
\text { avoided with } \\
\text { enoxaparin }\end{array}$ & Not reported & Enoxaparin \\
\hline \multicolumn{7}{|c|}{ Low-molecular-weight heparins versus unfractionated heparin } \\
\hline Reference & $\begin{array}{l}\text { Patient } \\
\text { population }\end{array}$ & $\begin{array}{l}\text { Incremental } \\
\text { cost (USD) }\end{array}$ & $\begin{array}{l}\text { Incremental effects } \\
\text { (VTE avoided or life- } \\
\text { years or QALYS } \\
\text { gained) }\end{array}$ & $\begin{array}{l}\text { ICER (USD/VTE event } \\
\text { avoided or life-years } \\
\text { or QALYS gained) }\end{array}$ & Bleeding complications & $\begin{array}{l}\text { Most } \\
\text { economically } \\
\text { attractive } \\
\text { drug }\end{array}$ \\
\hline $\begin{array}{l}\text { *Drummond, } \\
\text { 1994, } \\
\text { enoxaparin }\end{array}$ & HFS & $\begin{array}{l}\text { Net saving } \\
(\$ 43,609) \text { per } \\
1,000 \text { patients } \\
\text { with enoxaparin }\end{array}$ & $\begin{array}{l}\text { Four deaths avoided } \\
\text { per } 1,000 \text { patients with } \\
\text { enoxaparin }\end{array}$ & Enoxaparin dominant & Not reported & Enoxaparin \\
\hline $\begin{array}{l}\text { *Hawkins, 1997, } \\
\text { enoxaparin }\end{array}$ & THR & $\begin{array}{l}\$ 57,972 \text { per } \\
1,000 \text { patients } \\
\text { with enoxaparin }\end{array}$ & $\begin{array}{l}47 \text { DVT events avoided } \\
\text { per } 1,000 \text { patients with } \\
\text { enoxaparin }\end{array}$ & $\begin{array}{l}\$ 1,180 \text { per VTE event } \\
\text { avoided with } \\
\text { enoxaparin }\end{array}$ & $\begin{array}{l}\text { Not reported (implied } \\
\text { enoxaparin increased bleeding } \\
\text { risk) }\end{array}$ & Enoxaparin \\
\hline $\begin{array}{l}\text { Marchetti, 1999, } \\
\text { enoxaparin }\end{array}$ & THR & $\begin{array}{l}\text { Net saving } \\
(\$ 90,000) \text { per } \\
1,000 \text { patients } \\
\text { with enoxaparin }\end{array}$ & $\begin{array}{l}70 \text { life-years gained per, } \\
1000 \text { patients with } \\
\text { enoxaparin }\end{array}$ & Enoxaparin dominant & Not reported & Enoxaparin \\
\hline $\begin{array}{l}{ }^{*} \text { Etchells, 1999, } \\
\text { enoxaparin }\end{array}$ & $\begin{array}{l}\text { Colorectal } \\
\text { surgery }\end{array}$ & $\begin{array}{l}\$ 180,641 \text { per } \\
1,000 \text { patients } \\
\text { with enoxaparin }\end{array}$ & $\begin{array}{l}0 \text { VTE events avoided } \\
\text { with enoxaparin }\end{array}$ & UFH dominant & $\begin{array}{l}12 \text { additional major bleeding } \\
\text { events with enoxaparin }\end{array}$ & UFH \\
\hline $\begin{array}{l}\text { Lloyd, 2001, } \\
\text { enoxaparin }\end{array}$ & Medical & $\begin{array}{l}\text { Net saving } \\
(\$ 850) \text { per 1,000 } \\
\text { patients with } \\
\text { enoxaparin }\end{array}$ & $\begin{array}{l}21 \text { VTE events avoided } \\
\text { per 1,000 patients with } \\
\text { enoxaparin }\end{array}$ & Enoxaparin dominant & $\begin{array}{l}18 \text { fewer major bleeding } \\
\text { events with Enoxaparin }\end{array}$ & Enoxaparin \\
\hline $\begin{array}{l}\text { *Offord, 2003, } \\
\text { enoxaparin }\end{array}$ & Medical & $\begin{array}{l}\text { Net saving } \\
(\$ 54,649) \text { per } \\
1,000 \text { patients } \\
\text { with Enoxaparin }\end{array}$ & $\begin{array}{l}20.5 \text { VTE events and } 0.5 \\
\text { deaths avoided per } \\
\text { 1,000 patients with } \\
\text { enoxaparin }\end{array}$ & Enoxaparin dominant & Not reported & Enoxaparin \\
\hline $\begin{array}{l}\text { *McGarry, 2004, } \\
\text { enoxaparin }\end{array}$ & Medical & $\begin{array}{l}\$ 14,459 \text { per } \\
1,000 \text { patients } \\
\text { with enoxaparin }\end{array}$ & $\begin{array}{l}10 \text { VTE events and } 4.4 \\
\text { deaths avoided per } \\
\text { 1,000 patients with } \\
\text { enoxaparin }\end{array}$ & $\begin{array}{l}\$ 1,445 \text { per VTE event } \\
\text { avoided, and } \$ 10,360 \\
\text { per death avoided } \\
\text { with enoxaparin }\end{array}$ & $\begin{array}{l}\text { 2.7\% fewer bleeding events, } \\
0.9 \% \text { fewer episodes of HIT }\end{array}$ & Enoxaparin \\
\hline $\begin{array}{l}\text { *Schadlich, } \\
2006, \\
\text { enoxaparin }\end{array}$ & Medical & $\begin{array}{l}\text { Net saving } \\
(\$ 46,499) \text { per } \\
1,000 \text { patients } \\
\text { with Enoxaparin }\end{array}$ & $N / R$ & Enoxaparin dominant & $\begin{array}{l}7.7 \text { fewer major bleeding } \\
\text { episodes with enoxaparin }\end{array}$ & Enoxaparin \\
\hline
\end{tabular}


Table 3 Incremental costs, effects, and cost-efficacy ratios for the different modes of venous thromboembolism prophylaxis (Continued)

\begin{tabular}{|c|c|c|c|c|c|c|}
\hline $\begin{array}{l}\text { *Deitelzweig, } \\
2008\end{array}$ & Medical & $\begin{array}{l}\text { Net saving } \\
(\$ 339,361) \text { per } \\
1,000 \text { patients } \\
\text { with enoxaparin }\end{array}$ & $\begin{array}{l}11 \text { VTE events, three } \\
\text { deaths avoided per } \\
\text { 1,000 patients with } \\
\text { enoxaparin }\end{array}$ & Enoxaparin dominant & $\begin{array}{l}\text { Five major bleeding events, } \\
\text { four episodes of HIT avoided } \\
\text { per } 1,000 \text { patients with } \\
\text { enoxaparin }\end{array}$ & Enoxaparin \\
\hline $\begin{array}{l}\text { Wade, 2008, } \\
\text { enoxaparin }\end{array}$ & $\begin{array}{l}\text { Gynecology } \\
\text { oncology } \\
\text { Surgery }\end{array}$ & $\begin{array}{l}\text { Net saving } \\
(\$ 36,197) \text { per } \\
1,000 \text { patients } \\
\text { with enoxaparin }\end{array}$ & $\begin{array}{l}\text { Eight DVTs, } 18 \text { PE } \\
\text { events avoided per } \\
\text { 1,000 patients with } \\
\text { enoxaparin }\end{array}$ & Enoxaparin dominant & $\begin{array}{l}21 \text { additional major bleeding } \\
\text { events per 1,000 patients with } \\
\text { enoxaparin }\end{array}$ & Enoxaparin \\
\hline \multirow[t]{2}{*}{$\begin{array}{l}\text { *Lloyd, 1997, } \\
\text { nadroparin }\end{array}$} & Orthopedics & $\begin{array}{l}\text { Net savings } \\
(\$ 192,000) \text { per } \\
1,000 \text { patients } \\
\text { with enoxaparin }\end{array}$ & $\begin{array}{l}50 \text { VTE events avoided } \\
\text { per } 1,000 \text { patients with } \\
\text { enoxaparin }\end{array}$ & Enoxaparin dominant & Not reported & Nadroparin \\
\hline & $\begin{array}{l}\text { General } \\
\text { surgery }\end{array}$ & $\begin{array}{l}\text { Net savings } \\
(\$ 33,000) \text { per } \\
1,000 \text { patients } \\
\text { with enoxaparin }\end{array}$ & $\begin{array}{l}\text { Nine VTE events } \\
\text { avoided per 1,000 } \\
\text { patients with } \\
\text { enoxaparin }\end{array}$ & Nadroparin dominant & Not reported & Nadroparin \\
\hline $\begin{array}{l}\text { Heerey, 2005, } \\
\text { dalteparin }\end{array}$ & $\begin{array}{l}\text { General } \\
\text { surgery }\end{array}$ & $\begin{array}{l}\$ 473,000 \text { per } \\
1,000 \text { patients } \\
\text { with dalteparin }\end{array}$ & $\begin{array}{l}21 \text { QALYs per 1,000 } \\
\text { patients with dalteparin }\end{array}$ & $\begin{array}{l}\$ 20,337 / \text { QALY gained } \\
\text { with dalteparin }\end{array}$ & Not reported & Dalteparin \\
\hline \multicolumn{7}{|c|}{ Low-molecular-weight heparins versus warfarin } \\
\hline Reference & $\begin{array}{l}\text { Patient } \\
\text { population }\end{array}$ & $\begin{array}{l}\text { Incremental } \\
\text { cost (USD) }\end{array}$ & $\begin{array}{l}\text { Incremental effects } \\
\text { (VTE avoided or life- } \\
\text { years or QALYS } \\
\text { gained) }\end{array}$ & $\begin{array}{l}\text { ICER (USD/VTE event } \\
\text { avoided or life-years } \\
\text { or QALYS gained) }\end{array}$ & Bleeding complications & $\begin{array}{l}\text { Most } \\
\text { economically } \\
\text { attractive } \\
\text { drug }\end{array}$ \\
\hline $\begin{array}{l}\text { *O'Brien, 1994, } \\
\text { enoxaparin }\end{array}$ & THR & $\begin{array}{l}\$ 133,571 \text { per } \\
1,000 \text { patients } \\
\text { with LMWH }\end{array}$ & $\begin{array}{l}\text { Five VTE events, } 0.4 \\
\text { deaths avoided per } \\
1,000 \text { patients with } \\
\text { LMWH }\end{array}$ & $\begin{array}{l}\text { \$26,711 per VTE event } \\
\text { avoided, } \$ 334,055 \text { per } \\
\text { death avoided, } \\
\$ 32,158 \text { per life-year } \\
\text { gained with LMWH }\end{array}$ & Not reported & LMWH \\
\hline $\begin{array}{l}\text { *Menzin, 1995, } \\
\text { enoxaparin }\end{array}$ & THR & $\begin{array}{l}\$ 69,659 \text { per } \\
1,000 \text { patients } \\
\text { with LMWH }\end{array}$ & $\begin{array}{l}20.1 \text { VTE events and } 4.3 \\
\text { deaths avoided per } \\
1,000 \text { patients with } \\
\text { LMWH }\end{array}$ & $\begin{array}{l}\$ 3,466 \text { per VTE } \\
\text { avoided, } \$ 16,200 \text { per } \\
\text { additional death } \\
\text { avoided }\end{array}$ & Not reported & LMWH \\
\hline $\begin{array}{l}\text { *Hull, 1997, } \\
\text { tinzaparin }\end{array}$ & TKR, THR & $\begin{array}{l}\text { Net saving } \\
(\$ 52,690) \text { per } \\
1,000 \text { patients } \\
\text { with LMWH }\end{array}$ & $\begin{array}{l}60 \text { VTE events avoided } \\
\text { per } 1,000 \text { patients with } \\
\text { LMWH }\end{array}$ & LMWH dominant & $\begin{array}{l}2.2 \% \text { increase in major } \\
\text { bleeding events with } \mathrm{LMWH}\end{array}$ & LMWH \\
\hline $\begin{array}{l}\text { *Hawkins, 1998, } \\
\text { enoxaparin }\end{array}$ & TKR & $\begin{array}{l}\$ 126,766 \text { per } \\
1,000 \text { patients } \\
\text { with LMWH }\end{array}$ & $\begin{array}{l}145 \text { VTE events avoided } \\
\text { per 1,000 patients with } \\
\text { LMWH }\end{array}$ & $\begin{array}{l}\text { \$874 per VTE event } \\
\text { avoided with LMWH }\end{array}$ & $\begin{array}{l}0.3 \% \text { increased risk of major } \\
\text { bleeding event with } L M W H\end{array}$ & LMWH \\
\hline *Francis, 1999 & THR & $\begin{array}{l}\text { Net saving } \\
(\$ 153,000) \text { per } \\
1,000 \text { patients } \\
\text { treated with } \\
\text { LMWH }\end{array}$ & $\begin{array}{l}112 \text { VTE events avoided } \\
\text { per } 1,000 \text { patients with } \\
\text { LMWH }\end{array}$ & LMWH dominant & $\begin{array}{l}62 \text { more patients with bleeding } \\
\text { event with LMWH }\end{array}$ & LMWH \\
\hline $\begin{array}{l}\text { *Botteman, } \\
\text { 2002, } \\
\text { enoxaparin }\end{array}$ & THR & $\begin{array}{l}\$ 154,000 \text { per } \\
1,000 \text { patients } \\
\text { with LMWH }\end{array}$ & $\begin{array}{l}77 \text { DVTs avoided per } \\
1,000 \text { patients, } 40 \\
\text { QALYs gained per 1,000 } \\
\text { patients with LMWH }\end{array}$ & $\begin{array}{l}\text { \$2013 per DVT } \\
\text { avoided, \$40,169 per } \\
\text { death avoided, \$4349 } \\
\text { per QALY gained with } \\
\text { LMWH }\end{array}$ & Not reported & LMWH \\
\hline $\begin{array}{l}\text { Nerurkar, 2002, } \\
\text { enoxaparin }\end{array}$ & TKR & $\begin{array}{l}\text { Net saving }(\$ 1 \text {, } \\
054,000) \text { per } \\
1,000 \text { patients } \\
\text { with LMWH }\end{array}$ & $\begin{array}{l}\text { Seven deaths avoided } \\
\text { per } 1,000 \text { patients with } \\
\text { LMWH }\end{array}$ & LMWH dominant & Not reported & LMWH \\
\hline *Caprini, 2002 & THR & $\begin{array}{l}\$ 110,235 \text { per } \\
1,000 \text { patients } \\
\text { with LMWH }\end{array}$ & $\begin{array}{l}5.8 \text { VTE events avoided } \\
\text { per } 1,000 \text { patients with } \\
\text { LMWH }\end{array}$ & $\begin{array}{l}\$ 19,006 \text { per VTE event } \\
\text { avoided with LMWH }\end{array}$ & Not reported & LMWH \\
\hline \multicolumn{7}{|c|}{ Comparison of low-molecular-weight heparins and other agents } \\
\hline Reference & $\begin{array}{l}\text { Patient } \\
\text { population }\end{array}$ & $\begin{array}{l}\text { Incremental } \\
\text { cost (USD) }\end{array}$ & $\begin{array}{l}\text { Incremental effects } \\
\text { (VTE avoided or life- } \\
\text { years or QALYS } \\
\text { gained) }\end{array}$ & $\begin{array}{l}\text { ICER (USD/VTE event } \\
\text { avoided or life-years } \\
\text { or QALYS gained) }\end{array}$ & Bleeding complications & $\begin{array}{l}\text { Most } \\
\text { economically } \\
\text { attractive } \\
\text { drug }\end{array}$ \\
\hline
\end{tabular}


Table 3 Incremental costs, effects, and cost-efficacy ratios for the different modes of venous thromboembolism prophylaxis (Continued)

\begin{tabular}{|c|c|c|c|c|c|c|}
\hline $\begin{array}{l}\text { Levin, 2001, } \\
\text { desirudin versus } \\
\text { enoxaparin }\end{array}$ & THR & $\begin{array}{l}\$ 72,000 \text { per } \\
1,000 \text { patients }\end{array}$ & $\begin{array}{l}19.1 \text { life-years gained } \\
\text { per 1,000 patients with } \\
\text { desirudin }\end{array}$ & $\begin{array}{l}\$ 3,794 \text { per life-year } \\
\text { gained }\end{array}$ & Not reported & Desirudin \\
\hline $\begin{array}{l}\text { *Honorato, } \\
\text { 2004, bemiparin } \\
\text { versus } \\
\text { enoxaparin }\end{array}$ & TKR & $\begin{array}{l}\text { Net savings } \\
(\$ 227,000) \text { per } \\
1,000 \text { patients } \\
\text { with bemiparin }\end{array}$ & $\begin{array}{l}42 \text { VTE events avoided } \\
\text { per } 1,000 \text { patients with } \\
\text { bemiparin }\end{array}$ & Bemiparin dominant & Not reported & Bemiparin \\
\hline $\begin{array}{l}\text { *Attanasio, } \\
\text { 2001, dermatan } \\
\text { sulfate versus } \\
\text { UFH 5,000 U, } 3 \\
\text { times daily }\end{array}$ & $\begin{array}{l}\text { Surgical } \\
\text { cancer }\end{array}$ & $\begin{array}{l}\text { Net saving } \\
(\$ 53,000) \text { per } \\
1,000 \text { patients } \\
\text { with dermatan } \\
\text { sulfate }\end{array}$ & $\begin{array}{l}70 \text { DVTs avoided and } \\
3.1 \text { lives gained per } \\
1,000 \text { patients with } \\
\text { dermatan sulfate }\end{array}$ & $\begin{array}{l}\text { Dermatan sulfate } \\
\text { dominant }\end{array}$ & $\begin{array}{l}\text { Five additional major bleeding } \\
\text { events with dermatan sulfate }\end{array}$ & $\begin{array}{l}\text { Dermatan } \\
\text { sulfate }\end{array}$ \\
\hline $\begin{array}{l}\text { Heerey, 2005, } \\
\text { dalteparin 2,500 } \\
\cup \text { versus } \\
\text { dalteparin, } 5,000 \\
\cup\end{array}$ & $\begin{array}{l}\text { Abdominal } \\
\text { surgery }\end{array}$ & $\begin{array}{l}\$ 477,000 \text { per } \\
1,000 \text { patients } \\
\text { with dalteparin }\end{array}$ & $\begin{array}{l}18 \text { QALYs per } 1,000 \\
\text { patients with dalteparin }\end{array}$ & $\begin{array}{l}\$ 24,357 / \text { QALY gained } \\
\text { with dalteparin }\end{array}$ & Not reported & $\begin{array}{l}\text { Dalteparin } \\
5,000 \cup\end{array}$ \\
\hline $\begin{array}{l}\text { Wade, 2001, } \\
\text { tinzaparin } \\
\text { versus } \\
\text { enoxaparin }\end{array}$ & $\begin{array}{l}\text { Spinal cord } \\
\text { injury }\end{array}$ & $\begin{array}{l}\$ 223,259 \text { per } \\
1,000 \text { patients } \\
\text { with enoxaparin }\end{array}$ & Not reported & Not reported & Not reported & Not reported \\
\hline \multicolumn{7}{|c|}{ Fondaparinux versus enoxaparin } \\
\hline Reference & $\begin{array}{l}\text { Patient } \\
\text { population }\end{array}$ & $\begin{array}{l}\text { Incremental } \\
\text { cost (USD) }\end{array}$ & $\begin{array}{l}\text { Incremental effects } \\
\text { (VTE avoided or life- } \\
\text { years or QALYS } \\
\text { gained) }\end{array}$ & $\begin{array}{l}\text { ICER (USD/VTE event } \\
\text { avoided or life-years } \\
\text { or QALYS gained) }\end{array}$ & Bleeding complications & $\begin{array}{l}\text { Most } \\
\text { economically } \\
\text { attractive } \\
\text { drug }\end{array}$ \\
\hline *Gordois, 2003 & $\begin{array}{l}\text { THR, TKR, } \\
\text { HFS }\end{array}$ & $\begin{array}{l}\$ 10,000 \text { per } \\
1,000 \text { patients } \\
\text { by discharge } \\
\text { from hospital } \\
\text { with } \\
\text { fondaparinux }\end{array}$ & $\begin{array}{l}11 \text { VTE events, } 1.9 \\
\text { deaths avoided per } \\
1,000 \text { patients by } \\
\text { discharge from hospital } \\
\text { with fondaparinux }\end{array}$ & $\begin{array}{l}\$ 1,077 \text { per VTE event } \\
\text { avoided and } \$ 5,317 \\
\text { per death avoided } \\
\text { with fondaparinux }\end{array}$ & Not reported & Fondaparinux \\
\hline *Lundkvist, 2003 & $\begin{array}{l}\text { THR, TKR, } \\
\text { HFS }\end{array}$ & $\begin{array}{l}\text { Net saving } \\
(\$ 59,000) \text { per } \\
1,000 \text { patients } \\
\text { with } \\
\text { fondaparinux }\end{array}$ & $\begin{array}{l}17.9 \text { VTE events, } 2.6 \\
\text { deaths avoided per } \\
\text { 1,000 patients (average } \\
\text { among three } \\
\text { conditions) with } \\
\text { fondaparinux }\end{array}$ & $\begin{array}{l}\text { Fondaparinux } \\
\text { dominant }\end{array}$ & Not reported & Fondaparinux \\
\hline $\begin{array}{l}\text { Wade, 2003, } \\
\text { enoxaparin, } 30 \\
\text { mg twice daily } \\
\text { enoxaparin, } 40 \\
\text { mg once daily }\end{array}$ & THR & $\begin{array}{l}\text { Net savings } \\
(\$ 168,382) \text { per } \\
1,000 \text { patients } \\
\text { with enoxaparin }\end{array}$ & $\begin{array}{l}\text { Three VTE events per } \\
1,000 \text { patients with } \\
\text { enoxaparin }\end{array}$ & Enoxaparin dominant & $\begin{array}{l}27 \text { more bleeding episodes per } \\
1,000 \text { patients with } \\
\text { fondaparinux compared with } \\
\text { twice-daily enoxaparin Six } \\
\text { more bleeding episodes per } \\
\text { 1,000 patients with enoxaparin } \\
\text { once daily compared with } \\
\text { fondaparinux }\end{array}$ & $\begin{array}{l}\text { Enoxaparin } \\
\text { twice daily }\end{array}$ \\
\hline $\begin{array}{l}\text { Annemans, } \\
2004\end{array}$ & $\begin{array}{l}\text { THR, TKR, } \\
\text { HFS }\end{array}$ & $\begin{array}{l}\$ 2,800 \text { per } \\
1,000 \text { patients } \\
\text { with } \\
\text { fondaparinux }\end{array}$ & $\begin{array}{l}17.7 \text { VTE events per } \\
1,000 \text { patients with } \\
\text { fondaparinux }\end{array}$ & $\begin{array}{l}\text { \$158 per VTE event } \\
\text { avoided; } \$ 104 \text { per } \\
\text { death avoided with } \\
\text { fondaparinux }\end{array}$ & $\begin{array}{l}1.6 \text { more bleeding episodes per } \\
1,000 \text { patients with } \\
\text { fondaparinux }\end{array}$ & Fondaparinux \\
\hline $\begin{array}{l}\text { *Dranitsaris, } \\
2004\end{array}$ & $\begin{array}{l}\text { THR, TKR, } \\
\text { HFS }\end{array}$ & $\begin{array}{l}\text { Net saving } \\
(\$ 50,000) \text { per } \\
1,000 \text { patients } \\
\text { with } \\
\text { fondaparinux }\end{array}$ & $\begin{array}{l}16 \text { VTE avoided per } \\
1,000 \text { patients with } \\
\text { fondaparinux }\end{array}$ & $\begin{array}{l}\text { Fondaparinux } \\
\text { dominant }\end{array}$ & $\begin{array}{l}10 \text { more major bleeding events } \\
\text { per } 1,000 \text { patients with } \\
\text { fondaparinux }\end{array}$ & Fondaparinux \\
\hline Spruill, 2004 & $\begin{array}{l}\text { TKR (2002 } \\
\text { USD) }\end{array}$ & $\begin{array}{l}\text { Net saving } \\
(\$ 43,549) \text { per } \\
1,000 \text { patients } \\
\text { with } \\
\text { fondaparinux }\end{array}$ & $\begin{array}{l}36 \text { VTE events avoided } \\
\text { per 1,000 patients with } \\
\text { fondaparinux }\end{array}$ & $\begin{array}{l}\text { Fondaparinux } \\
\text { dominant }\end{array}$ & $\begin{array}{l}10 \text { more major bleeds and } \\
\text { three more minor bleeding } \\
\text { events per } 1,000 \text { patients with } \\
\text { fondaparinux }\end{array}$ & Fondaparinux \\
\hline
\end{tabular}


Table 3 Incremental costs, effects, and cost-efficacy ratios for the different modes of venous thromboembolism prophylaxis (Continued)

\begin{tabular}{|c|c|c|c|c|c|c|}
\hline Spruill, 2004 & $\begin{array}{l}\text { THR (2002 } \\
\text { USD) }\end{array}$ & $\begin{array}{l}\text { Net saving } \\
(\$ 18,898) \text { per } \\
1,000 \text { patients } \\
\text { with } \\
\text { fondaparinux }\end{array}$ & $\begin{array}{l}20 \text { VTE events avoided } \\
\text { per } 1,000 \text { patients with } \\
\text { fondaparinux }\end{array}$ & $\begin{array}{l}\text { Fondaparinux } \\
\text { Dominant }\end{array}$ & $\begin{array}{l}19 \text { more major bleeding events } \\
\text { per } 1,000 \text { patients with } \\
\text { fondaparinux }\end{array}$ & Fondaparinux \\
\hline Wade, 2004 & HFS & $\begin{array}{l}\$ 21,171 \text { per } \\
1,000 \text { patients } \\
\text { with } \\
\text { fondaparinux }\end{array}$ & $\begin{array}{l}34 \text { VTE events avoided } \\
\text { per } 1,000 \text { patients with } \\
\text { fondaparinux }\end{array}$ & $\begin{array}{l}\$ 623 \text { per VTE avoided, } \\
\$ 32,144 \text { per QALY } \\
\text { gained with } \\
\text { fondaparinux }\end{array}$ & $\begin{array}{l}\text { Approximately } 20 \% \text { increased } \\
\text { bleeding costs for fondaparinux }\end{array}$ & Fondaparinux \\
\hline *Sullivan, 2004 & $\begin{array}{l}\text { THR, TKR, } \\
\text { HFS }\end{array}$ & $\begin{array}{l}\text { Net savings } \\
(\$ 67,000) \text { per } \\
1,000 \text { patients } \\
\text { treated with } \\
\text { fondaparinux }\end{array}$ & $\begin{array}{l}\text { 3.7 VTE events avoided } \\
\text { per } 1,000 \text { patients with } \\
\text { Fondaparinux }\end{array}$ & $\begin{array}{l}\text { Fondaparinux } \\
\text { dominant }\end{array}$ & $\begin{array}{l}\text { Two more bleeding events per } \\
1000 \text { patients with } \\
\text { Fondaparinux }\end{array}$ & Fondaparinux \\
\hline *Szucs, 2005 & $\begin{array}{l}\text { THR, TKR, } \\
\text { HFS }\end{array}$ & $\begin{array}{l}\text { Net savings } \\
(\$ 18,153) \text { per } \\
1,000 \text { patients } \\
\text { treated with } \\
\text { fondaparinux }\end{array}$ & $\begin{array}{l}8.1 \text { VTE events avoided } \\
\text { per 1,000 patients with } \\
\text { fondaparinux }\end{array}$ & $\begin{array}{l}\text { Fondaparinux } \\
\text { dominant }\end{array}$ & $\begin{array}{l}1.6 \text { more bleeding events per } \\
1,000 \text { patients with } \\
\text { fondaparinux }\end{array}$ & Fondaparinux \\
\hline *Bjorvatn, 2005 & $\begin{array}{l}\text { THR, TKR, } \\
\text { HFS }\end{array}$ & $\begin{array}{l}\$ 53,553 \text { per } \\
1,000 \text { patients } \\
\text { treated with } \\
\text { fondaparinux }\end{array}$ & $\begin{array}{l}7.2 \text { VTE events avoided } \\
\text { per } 1,000 \text { patients with } \\
\text { fondaparinux }\end{array}$ & $\begin{array}{l}\$ 753 \text { per VTE avoided, } \\
\$ 6,782 \text { per death } \\
\text { avoided with } \\
\text { fondaparinux }\end{array}$ & $\begin{array}{l}\text { Two more bleeding events per } \\
1,000 \text { patients treated with } \\
\text { fondaparinux }\end{array}$ & Fondaparinux \\
\hline \multicolumn{7}{|c|}{ Dabigatran versus rivaroxaban and low-molecular-weight heparins } \\
\hline \multirow[t]{2}{*}{ Wolowacz, 2009} & THR & $\begin{array}{l}\text { THR Net savings } \\
(\$ 103,050) \text { per } \\
1,000 \text { patients } \\
\text { treated with } \\
\text { dabigatran }\end{array}$ & $\begin{array}{l}\text { Two VTEs avoided, } \\
\text { eight life-years, six } \\
\text { QALYs gained per 1,000 } \\
\text { patients treated with } \\
\text { dabigatran }\end{array}$ & Dabigatran dominant & $\begin{array}{l}\text { Five additional major bleeding } \\
\text { events, two episodes HIT } \\
\text { avoided per 1,000 patients } \\
\text { treated with dabigatran }\end{array}$ & Dabigatran \\
\hline & TKR & $\begin{array}{l}\text { Net savings } \\
(\$ 8,162) \text { per } \\
1,000 \text { patients } \\
\text { treated with } \\
\text { dabigatran }\end{array}$ & $\begin{array}{l}\text { Four VTEs avoided, } 9 \\
\text { life-years, } 7 \text { QALYs } \\
\text { gained per 1,000 } \\
\text { patients treated with } \\
\text { dabigatran }\end{array}$ & Dabigatran dominant & $\begin{array}{l}\text { Six additional major bleeding } \\
\text { events, two episodes HIT } \\
\text { avoided per 1,000 patients } \\
\text { treated with dabigatran }\end{array}$ & \\
\hline \multirow[t]{2}{*}{ McCullagh, 2009} & THR & $\begin{array}{l}\text { Net savings } \\
(\$ 24,104) \text { per } \\
1,000 \text { patients } \\
\text { treated with } \\
\text { rivaroxaban }\end{array}$ & $\begin{array}{l}7 \text { Life-years, } 10 \text { QALYS } \\
\text { gained per 1,000 } \\
\text { patients with } \\
\text { rivaroxaban }\end{array}$ & Rivaroxaban dominant & Not reported & Rivaroxaban \\
\hline & TKR & $\begin{array}{l}\text { Net savings } \\
(\$ 213,452) \text { per } \\
1,000 \text { patients } \\
\text { treated with } \\
\text { rivaroxaban }\end{array}$ & $\begin{array}{l}7 \text { Life-years, } 12 \text { QALYS } \\
\text { gained per 1,000 } \\
\text { patients with } \\
\text { rivaroxaban }\end{array}$ & Rivaroxaban dominant & & \\
\hline
\end{tabular}

HFS, hip-fracture surgery; ICER, incremental cost-efficacy ratio; LMWH, low-molecular-weight heparin; THR, total hip replacement; UFH, unfractionated heparin; USD, United States dollars; VTE, venous thromboembolism. *Industry-sponsored study.

Of the economic evaluations included in this review, 25 (64\%) received funding by manufacturers of one of the comparators. The "new" agent within the comparison was deemed to have a favorable (dominant or attractive incremental cost-effectiveness ratio) outcome in 38 (97.4\%) of the 39 economic evaluations ( $95 \% \mathrm{CI}, 86.5 \%$ to $99.9 \%$ ). Among the 25 studies funded by a pharmaceutical company, the sponsored medication was reported to be economically attractive in 24 (96.0\%) (95\% CI, $80.0 \%$ to $99.9 \%)$.

\section{Discussion}

In this systematic review of economic analyses of venous thromboembolism-prevention strategies in hospitalized patients, we found that all of the high-quality studies focused on pharmacologic prophylaxis. Low-molecularweight heparins were the most commonly studied "new" class of medication and were generally reported to be the dominant or economically attractive strategy in comparison with placebo, unfractionated heparin, or warfarin, among medical and surgical patients. However, among orthopedic patients, fondaparinux was favored over low-molecular-weight heparins. The two newest agents, dabigatran and rivaroxaban, are favored in the two most recent orthopedic surgery evaluations included in this review. Among the various strategies compared, the new agent had a favorable cost-efficacy ratio in $97 \%$ 


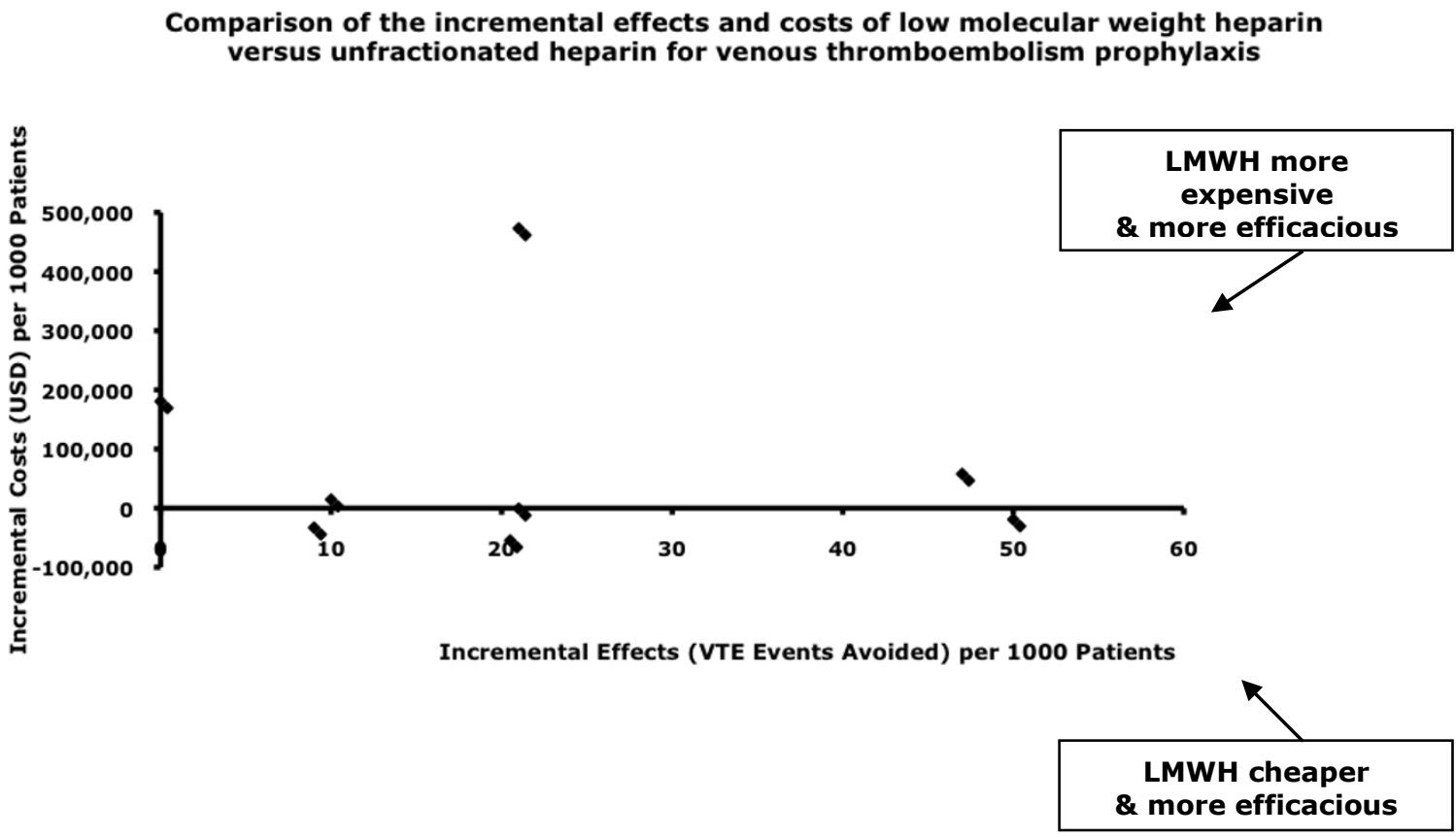

Figure 2 Comparison of the incremental effects and costs of low-molecular-weight heparin versus unfractionated heparin for venous thromboembolism prophylaxis.

of the studies, and most of these studies were sponsored by the manufacturers of the new agent.

Few systematic reviews of economic analyses comparing different modes of venous-thromboembolism prophylaxis in hospitalized patients have been conducted. In 1994, one such review of cost-efficacy analyses of low-molecular-weight heparins, unfractionated heparin, and warfarin in the prevention and treatment of venous thromboembolism in surgical patients, concluded that low-molecular-weight heparin was more efficacious and cost-effective after total hip arthroplasty [34]. Most recently, a trial comparing low-molecular-weight heparin

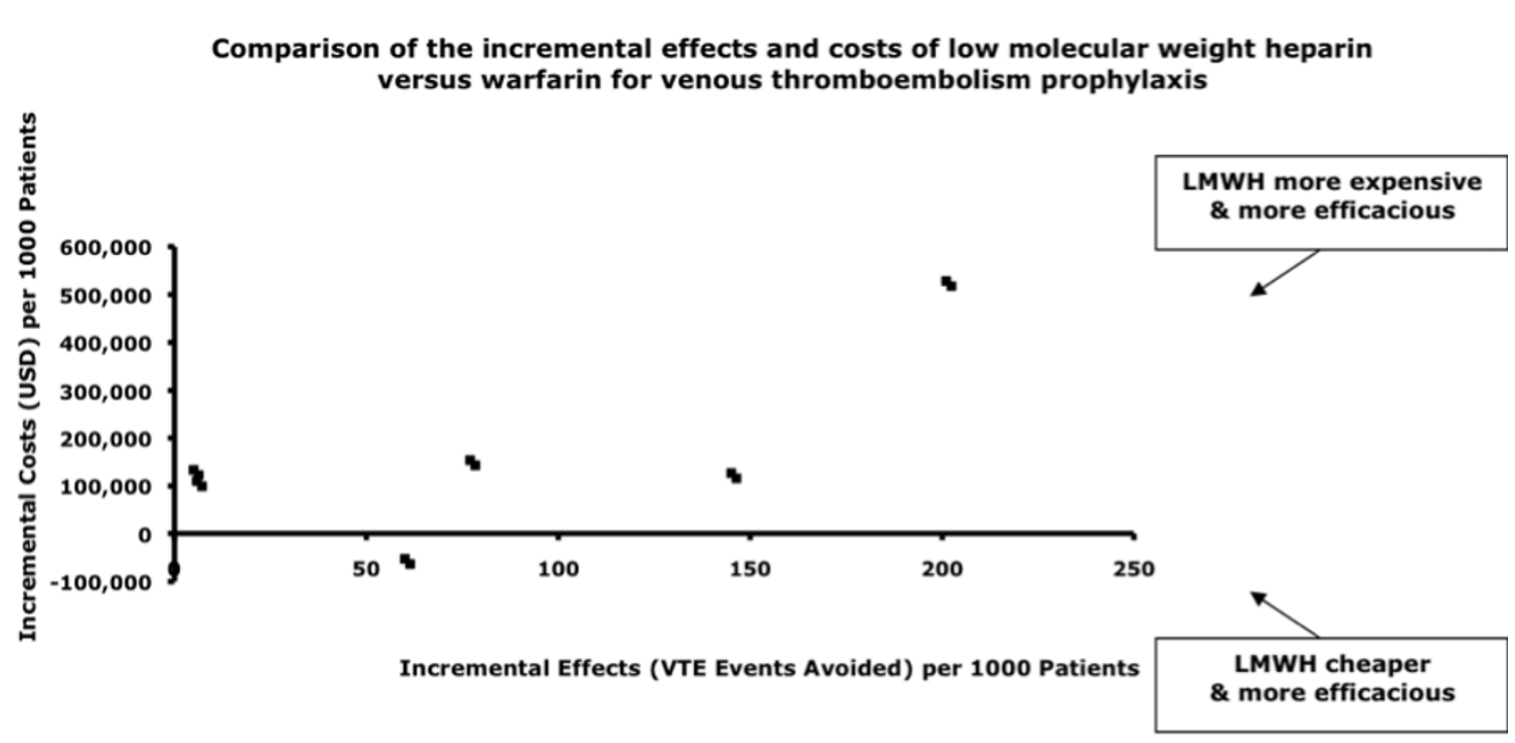

Figure 3 Comparison of the incremental effects and costs of low-molecular-weight heparin versus warfarin for venous thromboembolism prophylaxis. 


\section{Comparison of the incremental costs and effects of low molecular weight heparin} versus fondaparinux for venous thromboembolism prophylaxis

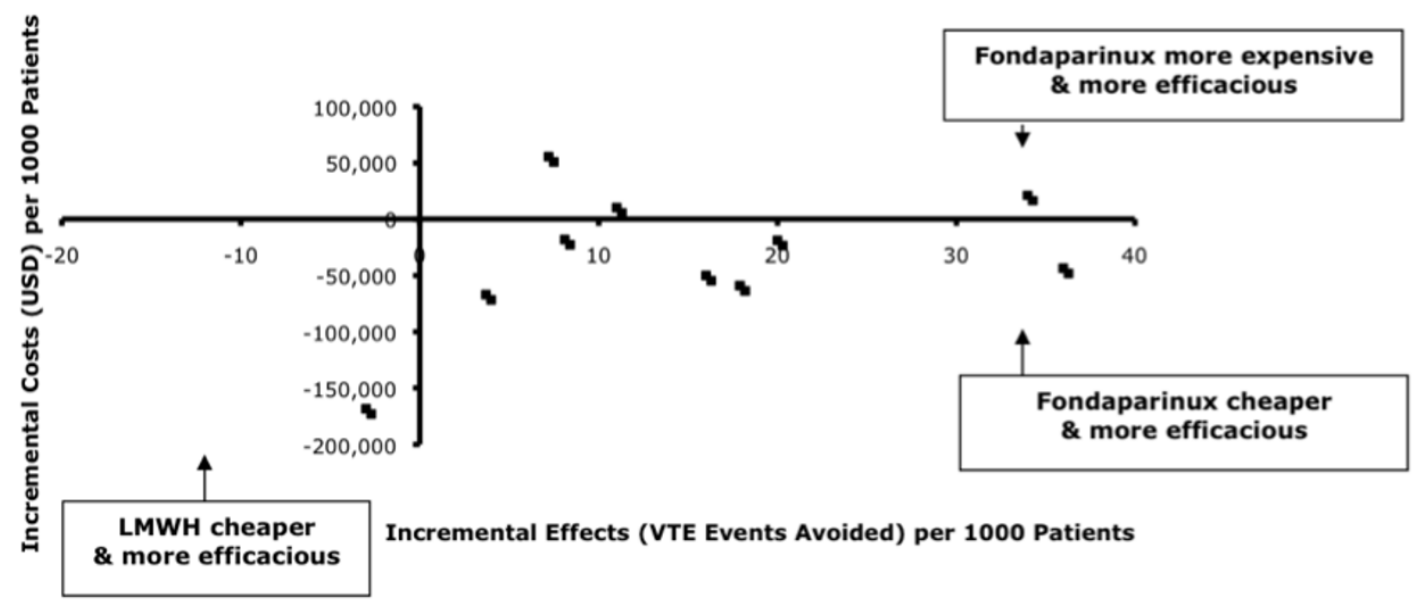

Figure 4 Comparison of the incremental effects and costs of low-molecular-weight heparin versus fondaparinux for venous thromboembolism prophylaxis.

with unfractionated heparin in hospitalized patients found low-molecular-weight heparin to be cost saving compared with unfractionated heparin and that lowmolecular-weight heparin was associated with a lower venous thromboembolism readmission rate at 30 and 90 days [59]. Our review includes 37 subsequently published analyses, focusing on both medical and surgical patient populations, and comparing newer pharmacologic agents for venous thromboembolism prophylaxis, such as fondaparinux, dabigatran, and rivaroxaban.

In this review, low-molecular-weight heparins appeared to offer superior prophylaxis efficacy compared with warfarin, unfractionated heparin, and placebo for orthopedic, general surgical, and medical patients. Fondaparinux was found to be economically more attractive for venous thromboembolism prevention compared with heparins because of greater efficacy in surgical and orthopedic patients, but may also be associated with increased bleeding. Among 11 economic analyses comparing enoxaparin with fondaparinux, all found that fondaparinux was economically attractive. More than half of these studies were either directly sponsored by the manufacturers of fondaparinux, or were based on original randomized controlled trials funded by the manufacturer.

Historically, many economic evaluations of new drugs have been sponsored by the drug manufacturer. However, this introduces the potential for bias in model construction and interpretation of the results. In a retrospective analysis of 107 trials in five leading medical journals with regard to outcome and sources of funding, studies sponsored by pharmaceutical companies were much less likely to favor traditional therapy over new drug treatment $[60,61]$. It is not surprising that new agents are incrementally efficacious; this is the nature of progress in medicine. However, new agents, typically still under patent protection, are virtually always substantially more expensive than comparator drugs. In our systematic review, 25 of the 39 studies were funded by pharmaceutical companies, and, with the exception of a single study [28], each of these found the sponsored drug more economically attractive than the comparator drug. Such consistency in incremental cost-effectiveness among more-expensive drugs is striking. Importantly, we could not detect a consistent bias in outcome between sponsored and nonsponsored evaluations; however, only a minority of evaluations did not receive sponsorship.

Strengths of our review include adherence to rigorous systematic review methods, which consisted of a comprehensive search strategy, broad eligibility criteria, and study selection by two independent adjudicators using $a$ priori criteria to minimize selection bias. Economic analyses are susceptible to investigator bias, often due to retrospective decision-model generation and retrospective acquisition of cost-and-effect data. To reduce this risk, we included only economic evaluations that incorporated outcome data from prospective randomized controlled trials. We conducted data abstraction and critical appraisal in duplicate, by using established criteria for assessing economic evaluations. We also addressed the relation of recency to market and for-profit 
sponsorship in influencing the reporting of economic evaluations. This review also has limitations. Many of the analyses within studies that we included come from a limited number of trials and cost-comparison models. For example, five studies used outcome data from the MEDENOX trial [20,22-24,33]. If most of the data are derived from a limited number of efficacy trials and cost models, similar results are likely to be found across economic evaluations. Although the studies included in this review received high ratings of internal validity, studies varied widely with respect to patient population, timehorizon of therapy, and payer perspective, making generalizability to other health care difficult. In addition, many evaluations rely on radiologic as opposed to clinical venous thromboembolism detection, which may overestimate the real-life clinical consequences of venous thromboembolism. Side effects of thromboprophylaxis may be underestimated, as randomized controlled trials often exclude patients at higher risk of bleeding. Furthermore, trials are generally underpowered to detect differences in rare drug-specific complications such as heparin-induced thrombocytopenia. This may lead to an overestimation of cost-efficacy, as reported in the economic evaluations in this review. Finally, our review included a predominance of orthopedic, general surgery, and medical patients, and thus, our findings may not generalizable to other patient populations.

Among economic analyses in this review, incremental cost-effectiveness ratios were commonly expressed in costs per venous thromboembolism events avoided, and they ranged from $\$ 500$ to $\$ 8,000$ per venous thromboembolism event avoided. These ranges are difficult to interpret, as no firmly established willingness-to-pay benchmarks exist for venous thromboembolism prevention. Costs per life-year or quality-adjusted life-year gained were less commonly reported, making economic comparisons of venous thromboembolism-prevention strategies and other interventions in healthcare similarly challenging. Comparing and combining ICERs performed with country-specific costs is challenging, as patient, disease, provider, and health-care system factors may influence transferability. We have not adjusted costs based on country-specific purchasing power parity but have adjusted based on changes in gross domestic product over time, and country-specific exchange rates [62].

An informative economic analysis should include both benefits and harms of interventions and the full associated costs over a relevant time horizon. Full costs of venous thromboembolism prophylaxis were not included in some studies. The common complications of venous thromboembolism prophylaxis include prophylaxis failure, leading to thrombotic events, bleeding, and heparin-induced thrombocytopenia [1]. All 39 studies evaluated in this systematic review accounted for breakthrough thrombotic events; however, only half included bleeding complications (Table 3), and none fully accounted for heparin-induced thrombocytopenia. The omission of these potentially serious complications may considerably affect the cost-comparison data of the individual studies. Most studies ascertained costs retrospectively and from the literature. This is often less complete or less accurate compared with prospective determination alongside a randomized controlled trial. Finally, none of the studies included in this review was prospectively designed before results of the randomized controlled trials were published; accordingly, they may be at risk for subjective decision-tree construction and interpretation.

Few studies have evaluated the cost-effectiveness of mechanical venous thromboembolism-prevention strategies, and none of the existing studies met our eligibility criteria. The UK National Institute for Health and Clinical Excellence have recommended considering graduated compression stockings in most patients, although economic evaluations of mechanical venous thromboembolism prophylaxis have generally been of low quality [63]. The paucity of rigorous evidence about the cost effectiveness of mechanical prophylaxis is striking. However, this may be explained by the fact that manufacturers of mechanical devices are often not required to furnish either effectiveness or cost data to regulatory bodies before their introduction and marketing.

\section{Conclusion}

In this systematic review of economic analyses of venous thromboembolism-prevention strategies in hospitalized patients, we found that low-molecular-weight heparins appear to be the most economically attractive strategy for venous thromboembolism prevention among the majority of medical and surgical patients, whereas fondaparinux is more economically attractive for orthopedic patients. The studies, however, may be at risk of overestimating efficacy and underestimating side effects such as bleeding. Approximately two thirds of all evaluations were directly funded by the manufacturer of the new drug, and such drugs were more likely to be found economically attractive in comparison to other strategies. Limited opportunity for peer-reviewed and independent funding for economic evaluations unfortunately leads to reliance on industry sponsorship in this field. In the future, we recommend that high-quality, prospective, cost-effectiveness analysis be planned alongside the intervention trials and that these be designed, conducted, analyzed, and reported independent of industry sponsors.

\section{Key messages}

- Low-molecular-weight heparins appear to be the most economically attractive strategy for venous 
thromboembolism prevention among the majority of medical and surgical patients, whereas fondaparinux is more economically attractive for orthopedic patients.

- However, approximately two thirds of all evaluations were directly funded by the manufacturer of the new drug.

- Such drugs were more likely to be found economically attractive in comparison to other strategies.

- Limited opportunity for peer-reviewed and independent funding for economic evaluations may lead to reliance on industry sponsorship and bias in this field.

\section{Additional material}

Additional file 1: Search Strategies and Results.

\begin{abstract}
Abbreviations
ACP: American College of Physicians; Cl: confidence interval; CIHI: Canadian Institute for Health Information; DARE: Database of Abstracts of Reviews of Effects; DRG: diagnosis-related group; HFS: hip-fracture surgery; ICER: incremental cost-efficacy ratio; LMWH: low-molecular-weight heparin; MEDENOX: prophylaxis in medical patients with enoxaparin trial; NICE: National Centre for Clinical Excellence; N/R: not reported; OHIP: Ontario Health Insurance Plan; RCT: randomized controlled trial; THR: total hip replacement; TKR: total knee replacement; UFH: unfractionated heparin; USD:
\end{abstract} United States dollars; VTE: venous thromboembolism.

\section{Acknowledgements}

We thank Michael Gould, Gordon Guyatt, Nicole Mittmann, Brian Chan, Peter Dodek, Peter Dodek, and the E-PROTECT collaborators, the Canadian Critical Care Trials Group, Heart and Stroke Canada, the University of Toronto Connaught New Staff Matching Fund, Canadian Intensive Care Foundation, and the Ontario Ministry of Health and Long-term Care Career Scientist Program.

\section{Author details}

'Department of Medicine, University of Toronto, Toronto, Ontario, Canada. ${ }^{2}$ Department of Critical Care Medicine, Sunnybrook Health Sciences Centre, University of Toronto, Toronto, Ontario, Canada. ${ }^{3}$ Department of Medicine, St. Joseph's Hospital and McMaster University, Hamilton, Ontario, Canada. ${ }^{4}$ Department of Medicine, Sunnybrook Health Sciences Centre, University of Toronto, Toronto, Ontario, Canada.

\section{Authors' contributions}

Drs. F, T, P, C, and G have made substantial contributions to conception and design, acquisition of data, analysis, and interpretation of data. They drafted the submitted article and revised it critically for important intellectual content. They provided final approval of the version to be published.

\section{Authors' information}

Dr. Fowler is a Career Scientist of the Ontario Ministry of Health and Longterm Care and an incoming Clinician Scientist of Heart and Stroke Foundation of Canada. Dr. Cook holds a Canada Research Chair from the Canadian Institutes of Health Research.

\section{Competing interests}

Drs. Pinto and Thirugnanam have no conflicts to disclose. Dr. Fowler's department has received funding for contract research, over the past 5 years from Eli Lilly, Wyeth, Novartis, and Fugisawa. Drs. Cook, Fowler, and Geerts are the primary investigator and collaborators, respectively, on PROTECT (a randomized clinical trial of low-molecular-weight heparin versus unfractionated heparin for thromboprophylaxis in critically ill patients) and DIRECT (a multicenter observational study of low-molecular-weight heparin thromboprophylaxis in critically ill patients with renal impairment). Both are investigator initiated and peer funded, but DIRECT received additional funding from Pfizer, the producers of dalteparin. PROTECT and DIRECT received donations of funding-in-kind for study drug from Pfizer, the makers of dalteparin. The design, conduct, oversight, analysis, interpretation, and write-up of DIRECT was without any influence from Pfizer. PROTECT is ongoing (in the conduct phase); the same conditions hold. Dr. Geerts, over the past 5-year period, has had the following financial relationships that, in a general way, relate to the subject matter discussed in the article or presentation (research grants/support received: Sanofi-Aventis, Pfizer; Bayer Healthcare (pending); consultancies: Astra Zeneca, Bayer Healthcare, Boehringer Ingelheim, Bristol-Myers Squibb, Covidien, Eli Lilly,

GlaxoSmithKline, Leo, Pharma, Merck KGaA, Pfizer, Roche, and Sanofi Aventis; honoraria: Astra Zeneca, Calea, Eisai, Oryx Pharma, Pfizer, and Sanofi-Aventis).

Received: 3 October 2011 Revised: 11 October 2011

Accepted: 9 March 2012 Published: 9 March 2012

\section{References}

1. Geerts W, Pineo G, Heit J, Bergqvist D, Lassen MR, Colwell CW, Ray JG: Prevention of Venous Thromboembolism: The Seventh ACCP conference on Antithrombotic and Thrombolytic Therapy. Chest 2004, 126:338S-400S.

2. Cook D, Crowther M, Meade M, Douketis J: Prevalence, incidence, and risk factors for venous thromboembolism in medical-surgical intensive care unit patients. J Crit Care 2005, 12:309-313.

3. Kakkar V, Flanc C, Howe C: Natural history of postoperative deep vein thrombosis. Lancet 1969, 2:230-234.

4. Barritt $D$, Jordan S: Anticoagulant drugs in the treatment of pulmonary embolism: a controlled trial. Lancet 1960, 1:1309-1312.

5. Lindblad B, Eriksson A, Bergqvist D: Autopsy-verified pulmonary embolism in surgical department: analysis of the period from 1951 to $1988 . \mathrm{Br} J$ Surg 1991, 78:849-852.

6. Patel R, Cook DJ, Meade MO, Griffith LE, Mehta G, Rocker GM, Marshall JC, Hodder R, Martin CM, Heyland DK, Peters S, Muscedere J, Soth M, Campbell N, Guyatt GH, for the Burden of Illness in Venous Thrombo Embolism in Critical Care (BITEC) Study Investigators; Canadian Critical Care Trials Group: Burden of illness in venous thromboembolism in critical care: a multicenter observational study. J Crit Care 2005, 20:341-347.

7. Shojania KG, Duncan BW, McDonald KM, Wachter RM, Markowitz AJ, Evidence Report/Technology Assessment Number 43: Making health care safer: a critical analysis of patient safety practices: summary.[http://www. ahra.gov/clinic/ptsafety/summary.htm].

8. Olin G, Machlin R, Agency for Healthcare Research and Quality, Center for Cost and Financing Studies: Health care expenses in the US civilian population [http://www.meps.ahrq.gov/papers/rf_01-r035/statisticaltables. $\mathrm{htm}$.

9. O'Brien BJ, Heyland D, Richardson WS, Levine M, Drummond MF: User's guide to the medical literature: XIII, How to use an article on economic analysis of clinical practice: $B$, What are the results and will they help me in caring for my patients? JAMA 1997, 277:1802-1814.

10. Chau Q, Cantor S, Caramel E: Cost-effectiveness of the bird's nest filter for preventing pulmonary embolism among patients with malignant brain tumors and deep venous thrombosis of the lower extremities. Support Care Cancer 2003, 11:795-799.

11. Epstein N: Intermittent pneumatic compression stocking prophylaxis against deep venous thrombosis in anterior cervical spinal surgery: a prospective efficacy study in 200 patients and literature review. Spine 2005, 30:2538-2543.

12. Ramos R, Salem BI, De Pawlikowski MP, Coordes C, Eisenberg S, Leidenfrost R: The efficacy of pneumatic compression stockings in the prevention of pulmonary embolism after cardiac surgery. Chest 1996, 109:82-85.

13. Sing R, Smith C, Miles W, Messick WJ: Preliminary results of bedside inferior vena cava filter placement: safe and cost-effective. Chest 1998, 114:315-316.

14. Warwick D, Harrison J, Glew D, Mitchelmore A, Peters TJ, Donovan J: Comparison of the use of a foot pump with the use of low-molecularweight heparin for the prevention of deep-vein thrombosis after total 
hip replacement: a prospective, randomized trial. J Bone Joint Surg 1998, , 80B: $1158-1166$

15. Williams J, Palfrey S: Cost effectiveness and efficacy of below knee against above knee graduated compression stockings in the prevention of deep vein thrombosis. Phlebologie 1988, 41:809-811.

16. Woolson S: Intermittent pneumatic compression prophylaxis for proximal deep venous thrombosis after total hip replacement. J Bone Joint Surg 1996, 78:1735-1740.

17. Chiasson TC, Manns BJ, Stelfox HT: An economic evaluation of venous thromboembolism prophylaxis strategies in critically ill trauma patients at risk of bleeding. PLoS Med 2009, 6:e1000098.

18. Gross domestic product deflator inflation calculator. [http://cost.jsc.nasa. gov/inflateGDP.html].

19. Universal currency converter. [http://www.xe.com/ucc/].

20. Pechevis M, Detournay B, Pribil C, Fagnani F, Chalanson G: Economic evaluation of enoxaparin versus placebo for the prevention of venous thromboembolism in acutely ill medical patients. Value Health 2000, 3:389-396.

21. Lloyd AC, Anderson PM, Quinlan DJ, Bearne A: Economic evaluation of the use of enoxaparin for thromboprophylaxis in acutely ill medical patients. $J$ Med Econ 2001, 4:99-113.

22. Lamy A, Wang X, Kent R, Smith KM, Gafni A: Economic evaluation of the MEDENOX trial: a Canadian perspective: medical patients with enoxaparin. Can Respir J 2002, 9:169-177.

23. Offord R, Lloyd A, Anderson P, Bearne A: Economic evaluation of enoxaparin for the prevention of venous thrombosis in acutely ill medical patients. Pharm World Sci 2004, 26:214-220.

24. Schadlich P, Kentsch M, Weber M: Cost effectiveness of enoxaparin as prophylaxis against venous thromboembolic complications in acutely ill medical inpatients: modeling study from the hospital perspective in Germany. Pharmacoeconomics 2006, 24:571-591.

25. Drummond $M$, Aristides $M$, Davies $L$, Forbes C: Economic evaluation of standard heparin and enoxaparin for prophylaxis against deep vein thrombosis in elective hip surgery. Br J Surg 1994, 81:1742-1746.

26. Hawkins $D$, Langley $C$, Krueger $K$ : Pharmacoeconomic model of enoxaparin versus heparin for prevention of deep vein thrombosis after total hip replacement. Am J Health Syst Pharm 1997, 54:1185-1190.

27. Marchetti M, Liberato N, Ruperto N, Barosi G: Long-term cost-effectiveness of low molecular weight heparin versus unfractionated heparin for the prophylaxis of venous thromboembolism in elective hip replacement. Haematologica 1999, 84:730-737.

28. Etchells E, McLeod R, Geerts W, Barton P, Detsky AS: Economic analysis of low-dose heparin vs the low-molecular-weight heparin enoxaparin for prevention of venous thromboembolism after colorectal surgery. Arch Intern Med 1999, 159:1221-1228.

29. McGarry L, Thompson D, Weinstein M, Goldhaber SZ: Cost effectiveness of thromboprophylaxis with a low-molecular-weight heparin versus unfractionated heparin in acutely ill medical inpatients. Am J Manag Care 2004, 10:632-642.

30. Lloyd A, Aitken JA, Hoffmeyer UK, Kelso EJ, Wakerly EC, Barber ND: Economic evaluation of the use of nadroparin calcium in the prophylaxis of deep vein thrombosis and pulmonary embolism in surgical patients in Italy. Pharmacoeconomics 1997, 12:475-485.

31. Heerey A, Suri S: Cost effectiveness of dalteparin for preventing venous thromboembolism in abdominal surgery. Pharmacoeconomics 2005, 23:927-944.

32. Wade WE, Spruill WJ: Cost-effectiveness of dalteparin versus unfractionated heparin as venous thromboembolism prophylaxis in malignant gynecologic surgery. Am J Ther 2008, 15:512-515.

33. Deitelzweig SB, Becker R, Lin J, Benner J: Comparison of the two-year outcomes and costs of prophylaxis in medical patients at risk of venous thromboembolism. Thromb Haemost 2008, 100:810-820.

34. O'Brien B, Anderson D, Goeree R: Cost-effectiveness of enoxaparin versus warfarin prophylaxis against deep-vein thrombosis after total hip replacement. CMAJ 1994, 150:1083-1090.

35. Menzin J, Colditz GA, Regan MM, Richner RE, Oster G: Cost-effectiveness of enoxaparin vs low-dose warfarin in the prevention of deep-vein thrombosis after total hip replacement surgery. Arch Intern Med 1995, 155:757-764

36. Hull R, Raskob G, Pineo G: Subcutaneous low-molecular-weight heparin vs warfarin for prophylaxis of deep vein thrombosis after hip or knee implantation: an economic perspective. Arch Intern Med 1997, 157:298-303.

37. Hawkins D, Langley $P$, Krueger $K$ : A pharmacoeconomic assessment of enoxaparin and warfarin as prophylaxis for deep vein thrombosis in patients undergoing knee replacement surgery. Clin Ther 1998, 20:182-195.

38. Botteman M, Caprini J, Stephens J: Results of an economic model to assess the cost-effectiveness of enoxaparin, a low-molecular-weight heparin, versus warfarin for the prophylaxis of deep vein thrombosis and associated long-term complications in total hip replacement surgery in the United States. Clin Ther 2002, 24:1960-1986.

39. Nerurkar J, Wade W, Martin B: Cost/Death averted with venous thromboembolism prophylaxis in patients undergoing total knee replacement or arthroplasty. Pharmacotherapy 2002, 22:990-1000

40. Francis CW, Pleil AM, Reinhart SP: A pharmacoeconomic evaluation of low-molecular-weight heparin in patients after total hip-replacement surgery. $P$ and $T$ 1999, 24:136-145.

41. Caprini JA, Arcelus JI, Kudrna JC, Sehgal LR, Oyslender M, Maksimovic D, MacDougall A: Cost-effectiveness of venous thromboembolism prophylaxis after total hip replacement. Phlebology 2002, 17:126-133.

42. Levin L, Bergqvist D: Cost effectiveness of desirudin compared with a low molecular weight heparin in the prevention of deep vein thrombosis after total hip replacement surgery. Pharmacoeconomics 2001, 19:589-597.

43. Honorato J, Gómez-Outes A, Navarro-Quilis A, Martínez-González J, Rocha E, Planès A: Pharmacoeconomic analysis of bemiparin and enoxaparin as prophylaxis for venous thromboembolism in total knee replacement surgery. Pharmacoeconomics 2004, 22:885-894

44. Attanasio E, Russo P, Carunchio G, Caprino L: Dermatan sulfate versus unfractionated heparin for the prevention of venous thromboembolism in patients undergoing surgery for cancer: a cost-effectiveness analysis. Pharmacoeconomics 2001, 19:57-68.

45. Wade W, Spruill W: Cost comparison of tinzaparin versus enoxaparin as deep venous thrombosis prophylaxis in spinal cord injury: preliminary data. Blood Coagul Fibrinol 2001, 12:619-625.

46. Gordois A, Posnett J, Borris L: The cost-effectiveness of fondaparinux compared with enoxaparin as prophylaxis against thromboembolism following major orthopedic surgery. J Thromb Haemost 2003, 1:2167-2174.

47. Lundkvist J, Bergqvist D, Jonsson B: Cost-effectiveness of fondaparinux versus enoxaparin as venous thromboembolism prophylaxis in Sweden. Eur J Health Econ 2003, 4:254-262

48. Wade E, Spruill W, Leslie R: Cost analysis: fondaparinux versus preoperative and postoperative enoxaparin as venous thromboembolic event prophylaxis in elective hip arthroplasty. Am J Orthop 2003, 4:201-206.

49. Annemans L, Minjoulat-Rey M, De Knock M: Cost consequence analysis of fondaparinux versus enoxaparin in the prevention of venous thromboembolism after major orthopedic surgery in Belgium. Acta Clin Belg 2004, 59:346-357.

50. Dranitsaris G, Kahn S, Stumpo C: Pharmacoeconomic analysis of fondaparinux versus enoxaparin for the prevention of thromboembolic events in orthopedic surgery patients. Am J Cardiovasc Drugs 2004, 4:325-333.

51. Spruill W, Wade W, Leslie R: A cost analysis of fondaparinux versus enoxaparin in total knee arthroplasty. Am J Ther 2004, 11:3-8.

52. Spruill W, Wade W, Leslie R: Cost analysis of fondaparinux versus enoxaparin as venous thromboembolism prophylaxis in elective hip replacement surgery. Blood Coagul Fibrinol 2004, 15:539-543.

53. Wade W, Spruill W, Leslie R: Cost analysis of fondaparinux versus enoxaparin as venous thromboembolism prophylaxis in hip fracture surgery. Am J Ther 2004, 11:194-198.

54. Bjorvatn A, Kristiansen F: Fondaparinux sodium compared with enoxaparin sodium: a cost-effectiveness analysis. Am J Cardiovasc Drugs 2005, 5:121-130.

55. Sullivan SD, Davidson BL, Kahn SR, Muntz JE, Oster G, Raskob G: A costeffectiveness analysis of fondaparinux sodium compared with enoxaparin sodium as prophylaxis against venous thromboembolism: use in patients undergoing major orthopaedic surgery. Pharmacoeconomics 2004, 22:605-620.

56. Szucs TD, Kaiser WE, Mahler F, Gutzwiller F: Thromboembolic prophylaxis with fondaparinux in major orthopaedic surgery: outcomes and costs. Heart Drug 2005, 5:121-130. 
57. McCullagh L, Tilson L, Walsh C, Barry M: A cost-effectiveness model comparing rivaroxaban and dabigatran etexilate with enoxaparin sodium as thromboprophylaxis after total hip and total knee replacement in the Irish healthcare setting. Pharmacoeconomics 2009, 27:829-846.

58. Wolowacz SE, Roskell NS, Maciver F, Beard SM, Robinson PA, Plumb JM, Dolan G, Brenkel IJ: Economic evaluation of dabigatran etexilate for the prevention of venous thromboembolism after total knee and hip replacement surgery. Clin Ther 2009, 31:194-212.

59. Merli G, Ferrufiono C, Lin M, Hussein M, Battleman D: Hospital-based costs associated with venous thromboembolism treatment regimens. $J$ Thromb Haemost 1996, 6:1077-1086.

60. Kelly R, Cohen L, Semple R: Relationship between drug company funding and outcomes of clinical psychiatric research. Psychol Med 2006, 36:1647-1657.

61. Yaphe J, Edman R, Knishkowy B, Herman J: The association between funding by commercial interests and study outcome in randomized controlled drug trials. Family Prac 2001, 18:565-568.

62. Goeree R, Burke N, O'Reilly D, Manca A, Blackhouse G, Tarride JE: Transferability of economic evaluations: approaches and factors to consider when using results from one geographic area for another. Curr Med Res Opin 2007, 23:671-682.

63. Robb P, Freer J, Seddon J, Young J, for the National Institute for Health and Clinical Excellence: Venous thromboembolism prophylaxis guidelines, April 2007 [http://www.nice.org.uk].

doi:10.1186/cc11241

Cite this article as: Thirugnanam et al:: Economic analyses of venous thromboembolism prevention strategies in hospitalized patients: a systematic review. Critical Care 2012 16:R43.

\section{Submit your next manuscript to BioMed Central} and take full advantage of:

- Convenient online submission

- Thorough peer review

- No space constraints or color figure charges

- Immediate publication on acceptance

- Inclusion in PubMed, CAS, Scopus and Google Scholar

- Research which is freely available for redistribution

Submit your manuscript at www.biomedcentral.com/submit
Biomed Central 